1827 Human Papillomavirus in Ocular Squamous Lesions

PJ Kobalka, BS Korn, DO Kikkawa, JH Lin. University of California, San Diego, San Diego, CA; VA San Diego Healthcare System, San Diego, CA.

Background: Human Papillomavirus (HPV) is tightly associated with the development of cervical dysplasia and carcinoma, and more recently, HPV has been linked to squamous lesions at other sites, especially head and neck. The role of HPV in the development of squamous lesions of the eye is poorly understood and controversial. Here, we retrospectively surveyed squamous lesions identified in ocular biopsies, enucleations, and orbital exenterations performed at our institution and examined them for HPV infection via immunohistochemistry and PCR.

Design: The ocular lesions were first evaluated for $\mathrm{p} 16$ immunoreactivity since $\mathrm{p} 16$ is strongly induced by HPV infection. Cases with strong diffuse positivity for p16 were further assessed for HPV serotype expression by PCR genotyping (ARUP HPV panel). The squamous ocular lesions selected for this study included seborrheic keratoses (SKs), pterygiums, verruca vulgari (VVs), squamous papillomas, achrocordons, conjunctival intraepithelial neoplasms (CIN), and squamous cell carcinomas (SCCs). All lesions were reviewed histologically for additional features, including solar damage and dysplasia. The lesions were further subdivided by patient gender and age and analyzed for trends. Cases reviewed were from The University of California San Diego (UCSD) and VA San Diego Hospital in which tissue specimens were submitted to the eye pathology service. In total, we analyzed approximately 50 seborrheic keratoses, 30 pterygiums, 12 achrocordons, 10 CINs, 10 SCCs, 5 squamous papillomas, and 5 VVs. Results: As of this submission $85 \%$ of SKs (11 of 13) tested have shown p16 immunoreactivity; $67 \%$ of pterygiums ( 4 of 6 ) have been positive; $86 \%$ of CINs ( 6 of $7) ; 100 \%$ of VVs ( 2 of 2 ) and $100 \%$ of SCCs ( 4 of 4 ). There was no clear link between cases that were p16-reactive and clinical demographics including age, gender, and ethnicity in our samples. The ocular squamous lesions further analyzed by HPV PCR genotyping were all negative for high-risk subtypes.

Conclusions: Our findings revealed a surprisingly large prevalence of p16 immunoreactivity in nearly all squamous lesions at our institutions. No clear correlation was seen between the p16-positive ocular squamous lesions and patient age or gender or ethnicity. Surprisingly, these lesions were not positive for high-risk HPV variants. Our findings raise the possibility that other HPV variants may be involved in development of ocular squamous lesions.

1828 Evaluation of Surgical Margins for Treatment of Ocular Surface Squamous Neoplasia (OSSN)

L Schoenfield, S Pabon, A Singh. Ohio State University Wexner Medical Center, Columbus, OH; Case Western Reserve University, Cleveland, OH; Cleveland Clinic Foundation, Cleveland, $\mathrm{OH}$.

Background: Ocular surface squamous neoplasia (OSSN) encompasses squamous dysplasia (mild-severe, CIN 1-3) and invasive squamous cell carcinoma (SCC) involving the conjunctiva and cornea. A traditionally accepted management modality is widemargin (4-5 $\mathrm{mm}$ ) excisional biopsy followed by cryotherapy. The conjunctival defect is then closed using conjunctival or amniotic membrane graft. Additional treatment strategies include chemotherapy and immunotherapy either in conjunction with or in lieu of surgery. Removing more tissue than necessary can compromise ocular function, for example by causing limbal cell insufficiency. Thus a re-evaluation was deemed warranted

Design: With IRB approval, a retrospective analysis of all patients presenting to one clinician (AS) with clinical OSSN from 2004-2012 requiring surgical excision was conducted.

Results: There were 32 cases, two of whom had been treated previously with topical chemotherapy for chemoreduction. All lesions were excised with $1-2 \mathrm{~mm}$ clinical margins and treated with cryotherapy. The mean follow-up time was 24.4 months (range: 1-84 months). In 17 cases there was corneal involvement. Pathological distribution of the lesions included 25 non-invasive OSSN ( 1 with CIN1, 5 CIN2, and 19 CIN3 or severe dysplasia/squamous carcinoma in situ) and 7 invasive SCC. 11 cases (34\%) had positive pathological margins. At 1-year and 5-year follow-up, only 2 of 32 eyes $(6 \%)$ had evidence of recurrence, respectively. The cases had SCC in situ and microinvasive SCC; both had positive initial margins. 3 patients in the cohort expired from causes unrelated to ocular disease.

Conclusions: OSSN can be managed surgically with adjunct cryotherapy using smaller margins without compromising clinical outcome. This may improve anatomical results and minimize the morbidity of the treatment.

1829 DLX Homeobox Transcription Factor Regulation of the Brn3a Homeobox Gene during Vertebrate Retina Development

$Q$ Zhang, D Eisenstat. Western University, London, ON, Canada; University of Alberta, Edmonton, AB, Canada.

Background: Retinal degeneration and retinal malignancy account for the majority of vision loss diseases. Our current knowledge of the genes involved in the processes of neuroretinal proliferation and cell differentiation is limited. The $D l \times 1 / D l \times 2$ double knockout mouse has reduced numbers ( $33 \%$ fewer) of retinal ganglion cells (RGC), due to enhanced apoptosis. Brn $3 a$ and Brn $3 b$ are closely related members of the Class IV POU-domain gene family, and share similar expression patterns and play functionally interchangeable roles in retinal development. $B r n 3 b$ is transcriptionally activated by both DLX1 and DLX2. We hypothesized that Brn3a is a DLX transcriptional target during retinal development.

Design: Embryonic retinas were dissected and chromatin immunoprecipitation (ChIP) of this tissue was utilized to identify DLX proteins bound to specific DNA elements of the Brn3a gene. Electrophoretic mobility shift assays (EMSA) were used to confirm specificity of this binding. Luciferase reporter gene assays were performed to confirm functional effects of DLX binding on the Brn $3 a$ promoter. In utero retinal electroporation was used as an in vivo functional study of DLX on Brn3a expression.

Results: Only DLX2 but not DLX1 protein bound to the Brn 3 a promoter in vivo. Using EMSA, recombinant DLX2 bound to specific Brn $3 a$ promoter sequences and specific supershifted bands resulted from the addition of DLX2 antibody. DLX2 binding to the Brn $3 a$ promoter region activated transcription of a luciferase reporter gene in vitro. In utero retinal electroporation shows that ectopic DLX2 expression promotes Brn $3 a$ expression in vivo.

Conclusions: DLX2, but not DLX1, regulates Brn $3 a$ transcription by directly binding to the Brn $3 a$ promoter region.

1830 Unsuspected Epithelial Neoplasias Associated with Pterygium: A 20 Year Study

P Zoroquiain, F Ceballos, H Dietrich, P Logan, VBravo-Filho, MN Burnier, Jr.. McGill University, Montreal, QC, Canada.

Background: Pinguecula and pterygium $(\mathrm{p} / \mathrm{pt})$ are conjunctival lesions strongly related to sunlight exposure. The incidence of these lesions varies considerably according to geographic areas. Ocular surface squamous neoplasia(OSSN) represents a broad spectrum of lesions starting as dysplasia and evolving to invasive squamous cell carcinoma of the conjunctiva. The relationship between $\mathrm{p} / \mathrm{pt}$ and OSSN has been described for various geographical areas including Brisbane, Australia (9.8\%; very high UV index), Florida, USA (1.7\%; moderate to high UV index), and in Ontario, Canada $(0 \%$; low UV index). To the best of our knowledge this is the first study looking at a Quebec patient population(low-UV index).

Design: All cases between 1993 and 2013 with a clinical diagnosis of $\mathrm{p} / \mathrm{pt}$ from the Henry C. Witelson Ocular Pathology Lab, McGill University, Montreal were reviewed. The final pathological diagnosis and clinical information, including age and gender, were obtained. Epithelial neoplasias were classified as actinic keratosis(a precancerous lesion), conjunctival intra epithelial neoplasia with mild dysplasia(CIN I), moderate dysplasia(CIN II), severe dysplasia/carcinoma in Situ(CIN III), and invasive squamous cell, mucoepidermoid, or sebaceous carcinoma.

Results: Two hundred and five cases were diagnosed clinically as pterygium and 22 as pinguecula; $51.2 \%$ of patients were male and $48.8 \%$ were female. The average age at diagnosis was $53.4 \pm 15.54$. The incidence of epithelial neoplasia in these $\mathrm{p} / \mathrm{pt}$ cases was $6.65 \% ; 60 \%$ were female and $40 \%$ were male and the average age was $53.8 \pm 14.9$. When analysed separately the incidence of epithelial neoplasia in pterygium and pinguecula were $4.88 \%$ and $18,88 \%$ respectively. The average age were $53.07 \pm 15.24$ and $58.75 \pm 21,47$ respectively. The average age of the patients with epithelial neoplasia associated to $\mathrm{p} / \mathrm{pt}$ is similar to the patients without neoplasia $(\mathrm{p}>0.05)$. The same results are found when pterygium is analysed separately. Epithelial neoplasias in $\mathrm{p} / \mathrm{pt}$ were diagnosed as actinic keratosis $(6.67 \%)$, CIN I (66.67\%), CIN II (13.33\%), CIN III $(13.33 \%)$

Conclusions: In this series, most epithelial lesions associated with p/pt were CIN I. The relative high proportion of neoplasia in pinguecula in this study could be due to the fact that pingueculas are surgically removed only when they have an abnormal appearance. Caution is advised due to the relative high prevalence of neoplastic lesions even in low UV index areas. Our relatively high rate of dysplasia in a low prevalence disease area with a low UV index challenges the main cause of this disease. This finding should be evaluated in new studies.

\section{Pancreas and Biliary Tree}

$1831 \quad$ Mutational Profiling (MP) of Discarded Supernatant Fluid: Its Role in Enhancing the Diagnostic Accuracy of Pancreatic Fine Needle Aspirations (FNA)

GAlsaati, S Nguyen, N Haddad, MK Sidawy. MedStar Georgetown University Hospital, Washington, DC

Background: Endoscopic ultrasound-guided (EUS) FNA of pancreatic lesions is associated with false negative results. Ancillary and molecular tests are emerging as useful tools for improving diagnostic accuracy. The purpose of this study was to evaluate the role of MP in discarded supernatant fluid from FNA of solid pancreatic lesions with negative, non-diagnostic (ND) or atypical cytologic diagnoses.

Design: Our database was searched (July 2011-July 2013) for EUS-FNA of solid pancreatic lesions which had supernatant fluid submitted for possible MP. 110 FNA from 108 patients were identified. 74 (67\%) were diagnostic of pancreatic neoplasms and the remaining 36 were classified as negative, ND or atypical. Only 21 of these 36 cases had available surgical or clinical follow-up and were included in the study. A clinically validated panel that included KRAS oncogene and 16 microsatellite markers associated with pancreaticobiliary cancer was used for MP. The results were classified as positive or negative for malignancy based on the presence of at least one mutation in any of the markers

Results: Table 1 summarizes the FNA, MP and follow-up findings. FNA diagnoses were: 8 negative, $10 \mathrm{ND}$ and 3 atypical. Follow-up revealed 6 pancreatic carcinomas and 15 benign outcomes. The supernatant of all 21 patients had sufficient amounts of amplifiable DNA for analysis. MP was positive in 2/6 malignant cases (one ND and one atypical by FNA). All cases with negative outcomes showed no mutations. 


\begin{tabular}{|c|c|c|}
\hline FNA Dx & MP & Outcome \\
\hline $\mathrm{N}$ & $\mathrm{N}$ & Pancreatitis* \\
\hline $\mathrm{N}$ & $\bar{N}$ & Pancreatitis** \\
\hline $\mathrm{N}$ & $N$ & Pancreatitis* \\
\hline $\mathrm{N}$ & $N$ & Pancreatitis* \\
\hline $\mathrm{N}$ & $\mathrm{N}$ & Pancreatitis* \\
\hline $\mathrm{N}$ & $N$ & Pancreatitis* \\
\hline $\mathrm{N}$ & $N$ & Pancreatitis* \\
\hline $\mathrm{N}$ & $\bar{N}$ & Accessory spleen* \\
\hline ND & $N$ & Pancreatitis* \\
\hline ND & $N$ & Pancreatitis* \\
\hline $\mathrm{ND}$ & $\bar{N}$ & Pancreatitis* \\
\hline ND & $N$ & Pancreatitis* \\
\hline ND & $N$ & Pancreatitis* \\
\hline ND & $N$ & No mass* \\
\hline ND & $\bar{N}$ & No mass* \\
\hline ND & $N$ & Carcinoma** \\
\hline ND & $\mathrm{N}$ & Carcinoma** \\
\hline ND & P & Carcinoma* \\
\hline Atypical & P & Carcinoma* \\
\hline Atypical & $N$ & Carcinoma*** \\
\hline Atypical & N & Carcinoma** \\
\hline
\end{tabular}

$\mathrm{P}=$ Positive; $\mathrm{N}=$ Negative; $\mathrm{ND}=$ Non-diagnostic; ${ }^{*}$ Clinical; $* *$ Surgical: ***Autopsy

Conclusions: We conclude that discarded supernatant fluid, even in ND pancreatic FNA, contains sufficient DNA for MP. The presence of a mutation may enhance our ability to detect malignancy, particularly when FNA is ND or atypical. Absence of a mutation does not exclude malignancy but with a negative FNA and in the appropriate clinical context, MP can provide additional information to guide clinical management.

1832 Complex Tubular Type Intracholecystic Papillary Tubular Neoplasms (ICPN): Further Clinicopathologic and Molecular Characterization

$S$ Balci, P Bagci, N Dursun, K-T Jang, B Saka, N Ohike, JC Roa, T Tajiri, C Moreno, M Rossi, JXu, R Hruban, H Matthaei, K Fisher, P Terry, V Adsay. EU, Atlanta, GA; SMC, Seoul, Korea; SU, Tokyo, Japan; PUC, Santiago, Chile; TU, Tokyo, Japan; JHU, Baltimore, MD; UB, Bonn, Germany; UT, Knoxville, TN.

Background: Preinvasive (adenomatous/papillary) lesions of the gallbladder, $>1 \mathrm{~cm}$ (intracholecystic papillary tubular neoplasms; ICPNs), composed of small, nonmucinous tubules with complex architecture is a poorly characterized group.

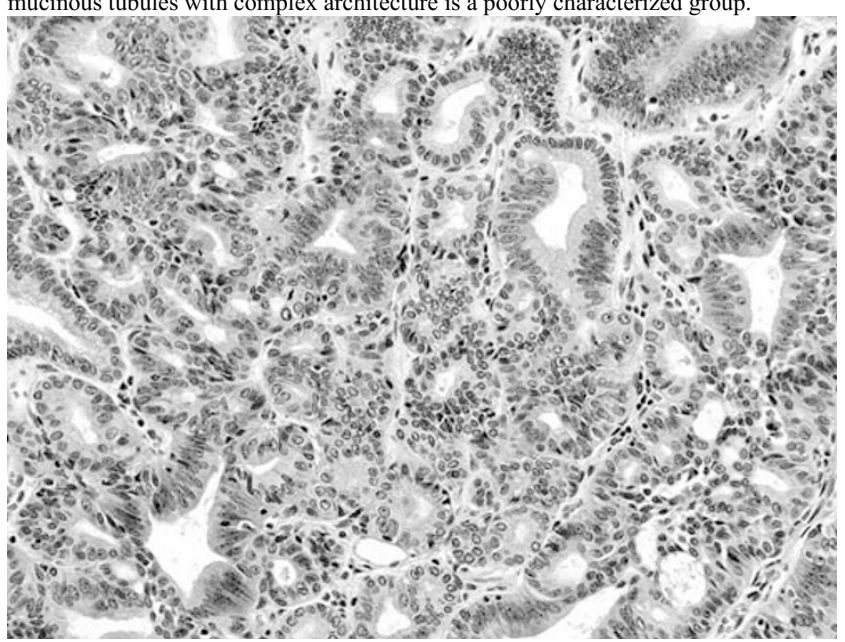

Design: Twenty examples were analyzed and contrasted with other GB pathology (Table). Four examples had been previously reviewed by 18 experts as a part of an international consensus, and all had been assigned a diagnosis of HGD/CIS (mostly "tubular/tubulopapillary carcinoma").

Results: They were pedunculated polyps with thin stalk and nodular growth, and mostly over-lined by normal epithelium (15), with overall architecture of cholesterol polyps. Some (6) also had cholesterol macrophages. Cystic glands with eosinophilic secretions and amorphous stromal hyalinization (5), morules (13), nuclei resembling papillary thyroid ca (13) were also seen. All revealed HGD $(<25 \%$ of the tumor in 3 ; $25-75 \%$ in 8 , and $>75 \%$ in 9 ). Uninvolved GB was devoid of any pathology, except 1 showing focal dysplasia. None had invasive carcinoma. Diffuse MUC6 staining seen (pyloric differentiation) in all, and focal MUC1 in 69\% (in high-grade areas); none had diffuse MUC2/CDX2. RNA sequencing performed in 4 revealed activation of various cancer related/cell movement gene groups.

\begin{tabular}{|c|c|c|c|c|c|c|c|}
\hline & $\begin{array}{l}\text { Complex } \\
\text { tubular } \\
\text { type } \\
\text { ICPNs } \\
(\mathrm{n}=20)\end{array}$ & \begin{tabular}{|l} 
Pyloric \\
gland \\
type \\
ICPNs \\
$(\mathrm{n}=13)$ \\
\end{tabular} & $\begin{array}{l}\text { All } \\
\text { ICPNs } \\
(n=123)\end{array}$ & $\begin{array}{l}\text { Cholesterol } \\
\text { polyps w } \\
\text { dysplasia } \\
(\mathrm{n}=13)\end{array}$ & $\begin{array}{l}\text { Cholesterol } \\
\text { polyps w/o } \\
\text { dysplasia } \\
(n=159)\end{array}$ & $\begin{array}{l}\text { Invasive } \\
\text { la } \\
(\mathrm{n}=655)\end{array}$ & $\begin{array}{l}\text { Cholecystitis } \\
(\mathrm{n}=1169)\end{array}$ \\
\hline Mean age & 51 & 65 & 61 & 61 & 49 & 66 & 50 \\
\hline $\mathrm{F} / \mathrm{M}$ & $9 / 10$ & $10 / 3$ & $80 / 38$ & $7 / 6$ & $102 / 51$ & $492 / 140$ & $823 / 333$ \\
\hline $\begin{array}{l}\text { Mean } \\
\text { tumor size } \\
\text { (cm) }\end{array}$ & 1.8 & 1.6 & 2.6 & 1.4 & 0.5 & 2.7 & N/A \\
\hline $\begin{array}{l}\text { Invasion } \\
\text { incidence }\end{array}$ & $0 / 20$ & $2 / 13$ & $68 / 123$ & $3 / 13$ & $0 / 159$ & N/A & N/A \\
\hline
\end{tabular}

Conclusions: ICPNs with complex tubular pattern of non-mucinous cells form a clinicopathologically distinct subset with a propensity to occur in men, in GBs without injury, and show association with cholesterol polyps. Although there is overt cyto-architectural complexity (HGD), and molecular analysis also shows activation of cancer related pathways; they seem to have virtually no ability to become invasive unlike other ICPNs.

\section{Medullary Carcinomas of the Ampulla: Clinicopathologic Analysis of 11 Cases}

S Balci, B Saka, P Bagci, K-T Jang, A Krasinskas, G Kim, NOhike, T Tajiri, O Basturk, $N$ Dursun, $N$ Zarrabi, J Knight, V Adsay. Emory, Atlanta, GA; SMC, Seoul, Korea; UCSF, San Francisco, CA; SU, Tokyo, Shinagawa, Japan; TU, Tokyo, Hiratsuka, Japan; MSKCC, NY, NY.

Background: Medullary carcinomas (MC) are now recognized as a distinct type of carcinoma in the GI tract. There is virtually no data on the frequency or clinicopathologic characteristics of the ampullary counterparts of these tumors.

Design: 359 ampullary carcinomas (AC) were analyzed. Non-ampullary duodenal as well as pancreatic and distal CBD carcinomas were carefully excluded.

Results: Eleven cases fulfilled the criteria for MC (3.2\% of ACs). MCs occurred in younger patients than other ACs (mean age, 57 vs $65 ; \mathrm{p}=0.03$ ), and more commonly in males $(\mathrm{M} / \mathrm{F}=9 / 2 \mathrm{vs} 197 / 144 ; \mathrm{p}=0.13)$. Size of invasive carcinoma was larger than that of other ACs (mean, 27.5 vs $18.5 \mathrm{~mm} ; \mathrm{p}=0.01$ ). Four $(40 \%)$ had positive lymph nodes (vs $46 \%$ ). In addition to classical medullary pattern (syncytial growth, pushingborder infiltration and peritumoral inflammation), 8 cases showed abortive gland-like formations within the syncytial nodules, and one also showed focal signet ring cells. Nine had separate minor mixed component of other carcinoma patterns: mucinous in 6 ; tubular in 4 (tubular-intestinal 1, tubular-pancreatobiliary 3); squamoid in 2; poorly differentiated, NOS in 2; acinar-like in 1; rhabdoid in 1. None revealed loss of INI-1 by immunohistochemistry. Preinvasive (adenomatous) component was either absent ( 8 cases) or if present ( 3 cases; intestinal in 2 , gastric-pancreatobiliary in 1 ), it was minimal in amount except for one case. Six had abundant intratumoral neutrophil infiltration and 4 had prominent eosinophils. As for site-specific origin in the ampulla, 8 tumors were AMP-NOS (papilla of Vater origin), 2 were AMP-duodenal (arising from the duodenal surface of the ampulla), and 1 was associated with intraampullary papillary-tubular neoplasm. MCs were strongly associated with microsatellite instability, with $7 / 8$ cases revealing loss of MMR proteins by immunohistochemistry (vs 11/86 non-medullary ACs; $\mathrm{p}<0.001$ ). Of these 7 cases, 6 had MLH1 and PMS2 losses, and 1 had MSH2 loss. Although the 3-yr and 5-yr survival rates of $67 \%$ and $67 \%$ appeared to be higher than those of other ACs $(61 \%$ and $47 \%)$, the difference did not reach statistical significance $(\mathrm{p}=0.47)$.

Conclusions: Medullary carcinomas constitute $3 \%$ of ACs. They occur predominantly in males, and in slightly younger age group. They are closely associated with microsatellite instability. Despite their large size and poorly differentiated appearance, their clinical behavior appears to be similar, if not better, than that of other ACs.

\section{Pitfalls of Frozen Ssction Interpretation for Pancreatectoy after Neoadjuvant Therapy}

AL Barbieri, S Tsai, DB Evans, K Oshima. Medical College of Wisconsin, Milwaukee, WI.

Background: Neoadjuvant chemoradiation therapy (NCRT) followed by pancreatectomy has been increasingly used in the treatment of pancreatic adenocarcinoma. Frozen sections play an important role in achieving complete resection in these cases. Our institution has a high volume of NCRT pancreatic resections and we are frequently faced with evaluating the margin status intraoperatively; potential pitfalls in the interpretation of these challenging specimens have not been previously described.

Design: We retrospectively reviewed the frozen section and permanent slides from 145 patients who completed NCRT and underwent pancreatic resection at our institution from 2009 to 2013; thirty-six patients who underwent pancreatectomy without neoadjuvant treatment were used as the control group. Frozen section diagnoses and final permanent diagnoses were recorded. Frequency and causes of discrepancy were analyzed.

Results: Three hundred and twenty-five frozen sections (183 pancreatic margins and 142 bile duct margins) were reviewed. Major discrepancies were seen in 8 cases within the NCRT group ( 5 false negatives and 3 false positives). All of the five false negative discrepancies occurred at the pancreatic margin and there were no false negatives at the bile duct margin. False positives were seen in both the pancreatic (2) and bile duct (1) margins. A single false positive was identified in the control group and there were no false negatives. Our preliminary data suggests that false negatives are a more frequent concern in the NCRT group. Based on our review, the NCRT tumors are inconspicuous and malignant cells are present in a diffusely fibrotic stroma without the focal stromal reaction is helpful in the evaluation of non-NCRT cases. Tumors can also be discontinuous after NCRT with positive margins despite a more prominent distant focus. We have also seen invasive carcinoma that can mimic PanIN.

Conclusions: Frozen section interpretation after NCRT is challenging, and the false negative rate is higher than the control group. Pathologists should be aware of the pitfalls after NCRT to improve precision and accuracy.

\section{The Histopathology of PRSS1 Hereditary Pancreatitis}

TL Bartholow, RK Pai, JA Kant, HJZeh, KK Lee, M Wijkstrom, D Yadav, RE Brand, JS Chennat, ME Lowe, GI Papachristou, A Slivka, DC Whitcomb, A Humar, AD Singhi. UPMC, Pittsburgh, PA.

Background: Hereditary pancreatitis is an autosomal dominant disorder with $80 \%$ penetrance and variable expressivity. The vast majority of cases have been linked to mutations within the cationic trypsinogen gene, also referred to as serine protease 1 (PRSS1). Other than inheritance, PRSSI pancreatitis has been considered clinically and pathologically indistinguishable from other etiologies of chronic pancreatitis. However, to date, the histologic findings of PRSS1 pancreatitis have not been well-described. 
Design: We collected pancreatic specimens from 10 PRSS1 patients of various ages and examined their clinicopathologic features. In addition, we compared these findings with more common etiologies of chronic pancreatitis including alcohol-associated and obstructive chronic pancreatitis.

Results: Patients at the time of resection ranged in age from 9 to 66 years (median, 29 years) with a slight female predominance $(60 \%)$. All patients reported a history of intermittent abdominal pain with an age of onset ranging from infancy to 21 years of age. Examination of the gross and microscopic findings suggested a sequential pattern of changes with increasing patient age. In pediatric patients $(n=4)$, although in most cases the pancreas was grossly normal, there was microscopic variation in lobular size and shape. While the central portions of the pancreas displayed parenchymal loss accompanied by loose perilobular and interlobular fibrosis, the periphery was remarkable for replacement by mature adipose tissue. These changes were more developed in younger adults $(n=2)$, where fatty replacement seemed to extend from the periphery to the central portions of the pancreas. With older patients $(n=4)$, the pancreas showed marked atrophy and extensive replacement by mature adipose tissue with scattered islets of Langerhans and rare acinar epithelium concentrated near the main pancreatic duct. In comparison, pancreata from patients with alcohol-associated $(n=14)$ and obstructive $(\mathrm{n}=8)$ chronic pancreatitis also demonstrated marked parenchymal loss, but replacement with prominent fibrosis.

Conclusions: In summary, contrary to previous assertions, $P R S S I$ hereditary pancreatitis is characterized by progressive lipomatous atrophy of the pancreas. As genetic testing for hereditary pancreatitis becomes more widespread, future studies should provide mechanistic insight into this debilitating disease.

1836 Combined Analysis of GNAS and KRAS Mutations in the Diagnosis of Pancreatic Mucinous Cysts

TL Bartholow, MN Nikiforova, A Khalid, KE Fasanella, K McGrath, RK Pai, NP Ohori, RE Brand, JS Chennat, GI Papachristou, A Slivka, HJ Zeh, AH Zureikat, KK Lee, A Tsung, GS Mantha, AD Singhi. UPMC, Pittsburgh, PA.

Background: The international consensus guidelines for the management of intraductal papillary mucinous neoplasms (IPMNs) and mucinous cystic neoplasms (MCNs) were recently updated in 2012. While there are a few major changes, appropriate treatment stratification relies on the assumption that mucinous cysts can be accurately distinguished from other cystic lesions of the pancreas. Previous studies using surgical resection material have identified recurrent mutations in GNAS and KRAS in pancreatic mucinous neoplasms, particularly IPMNs. Yet, the diagnostic utility of testing for both GNAS and KRAS mutations in pancreatic cyst fluid obtained by endoscopic ultrasoundfine needle aspiration remains unclear.

Design: Pancreatic cyst fluid DNA obtained by endoscopic ultrasound-fine needle aspiration was retrospectively collected from 75 pancreatic cysts that later underwent surgical resection. These cases included 36 IPMNs, 9 adenocarcinomas arising in association with an IPMN, 16 MCNs, 10 cystic pancreatic neuroendocrine tumors, 2 retention cysts, 1 pseudocyst and 1 lymphoepithelial cyst. In addition, 6 cyst fluid DNA specimens were selected that clinically corresponded to serous cystadenomas based on radiographic imaging, endoscopic ultrasound, CEA level of $<2.0 \mathrm{ng} / \mathrm{mL}$ and cytopathologic findings. All cases were analyzed for mutations in GNAS (codon 201) and $K R A S$ (codons 12,13 and 61).

Results: Point mutations in GNAS were detected in 13 of $36(36 \%)$ IPMNs, and 2 of $9(22 \%)$ adenocarcinomas arising in association with an IPMN. KRAS mutations were identified in $24(67 \%)$ IPMNs, $7(78 \%)$ adenocarcinomas, and 1 of $16(6 \%)$ MCNs. Mutations in either gene were present in 29 (89\%) IPMNs, 8 (89\%) adenocarcinomas, and $1(6 \%) \mathrm{MCN}$. No mutations were found in cyst fluid from cystic pancreatic neuroendocrine tumors, serous cystadenomas, retention cysts, a pseudocyst and a lymphoepithelial cyst. Overall, GNAS and KRAS mutations had a specificity of $100 \%$, but a sensitivity of $62 \%$ for mucinous differentiation. Among IPMNs, mutations in either gene had a specificity and sensitivity of $97 \%$ and $82 \%$, respectively.

Conclusions: In summary, the combination of GNAS and KRAS testing was highly specific and sensitive for IPMNs; however, should be interpreted as part of a multidisciplinary approach. Further, the lack of sensitivity for MCNs highlights the need for additional markers to improve the detection of pancreatic mucinous neoplasms.

1837 Pancreatic Acinar Cell Carcinomas with Prominent Neuroendocrine Differentiation: Clinicopathologic Analysis of a Distinct and Diagnostically Challenging Neoplasm

O Basturk, V Adsay, RH Hruban, Z Yang, TJ Giordano, C Shi, B Saka, DS Klimstra MSKCC, NY, NY; Emory, Atlanta, GA; JHU, Baltimore, MD; Hershey MC, Hershey, PA; University of Michigan, Ann Arbor, MI; Vanderbilt, Nashville, TN

Background: Although focal neuroendocrine(NE) differentiation is common in pancreatic acinar cell carcinomas (ACCs) and thus largely ignored, those with prominent NE component are recognized as mixed carcinomas (MANECs). Because of their rarity, MANECs are poorly characterized.

Design: The clinicopathologic features and survival of 28 MANECs were contrasted with those of 52 primary pancreatic poorly differentiated NECs (PDNECs).

Results: Twenty eight cases were identified (M:F=16/10; age, 27-85 yrs). Only 2 presented with jaundice. Tumors measured $1.5-27 \mathrm{~cm}$ (median, 6.9); were ill-defined, fleshy, and multilobulated. All 28 MANECs had evidence of both acinar (trypsin/ chymotrypsin) and NE (chromo/synapto) differentiation in at least $30 \%$ of the neoplastic cells. Two also had ductal differentiation (intracellular mucin/IHC labeling for glycoproteins). In 26 of the 28 MANECs, the ACC and NEC components merged intimately and individual neoplastic cells revealed dual differentiation. In fact, 15 cases were originally diagnosed as PDNEC and reclassified as MANEC only after IHC staining was performed. By IHC, $100 \%$ of the MANECs expressed chymotrypsin, $95 \%$ trypsin, $94 \%$ synapto, $80 \%$ chromo, and the NEC components had Ki67 indexes of 30\%->80\%.
None of the 6 cases tested had KRAS mutations. $74 \%$ of the patients had nodal and/ or distant metastases at presentation and additional $10 \%$ developed distant metastases subsequently. At last follow-up; 11 patients DOD; 13 were AWD. The median survival for all patients was 44 mos (range,1-129) with 1- and 3-yr survival rates of $79 \%$ and $56 \%$, respectively ( $v s 11 \mathrm{mos}$; and $44 \%$ and $21 \%$ for PDNECs; $\mathrm{p}=0.0035$ ).

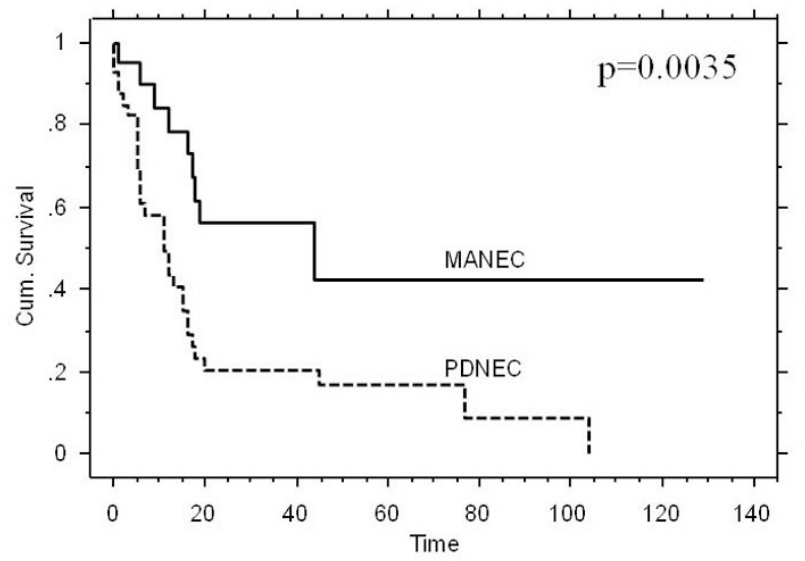

Conclusions: MANEC is often misdiagnosed as PDNEC. Although MANECs are also aggressive carcinomas, their survival seems to be significantly better than that of PDNECs. More importantly, MANECs and PDNECs are generally treated with different chemotherapy regimens after resection. Therefore, their distinction is of clinical importance and mandates thorough IHC evaluation for all poorly differentiated carcinomas of the pancreas.

1838 Endoscopic Ultrasound Guided Fine Needle Aspiration of Pancreatic Lesions: An Institution's Experience

JP Bergeron, KD Perry, PM Houser, J Yang. Medical University of South Carolina, Charleston, SC; University of Manitoba, Winnipeg, MB, Canada.

Background: Endoscopic ultrasound-guided fine needle aspiration (EUS-FNA) has emerged as the diagnostic modality of choice for mass lesions in the pancreas. The aim of this study was to determine the accuracy and pitfalls of EUS-FNA in the diagnosis of pancreatic lesions.

Design: Cases of EUS-FNA of pancreatic lesions performed from 2007 to mid of 2012 were retrieved from the department's database. The cases with histological follow up were selected and the accuracy of cytologic diagnoses was assessed using the histological diagnoses as the gold standard. In statistical analysis, cytologic diagnosis of Neoplastic, Suspicious, and Malignant were classified as positive. In one calculation, cytologic diagnosis of Atypical was included as positive while in another it was included as negative. The cases with cytologic-histological discrepancy were reviewed to identify sources of errors.

Results: A total of 1,212 cases from 1,104 patients (518 women, 586 men, and age ranging from 18 to 94 with average of 63.5 years) were identified. Cytologic diagnoses included 52 Unsatisfactory, 224 Benign, 129 Atypical, 140 Neoplasm, 35 Suspicious, and 632 Malignant. Of them, 396 patients had histological follow up information available and the cytologic-histological correlation is shown in Table 1. Sensitivity, specificity, PPV and NPV were $86.7 \%, 67.9 \%, 90.7 \%$, and $58.5 \%$, with Atypical diagnosis as positive, and $73.7 \%, 87.7 \%, 95.6 \%$, and $48.0 \%$, with Atypical as negative. The major difficulty in EUS-FNA cytology was to differentiate pancreatic mucinous neoplasms from contaminants of gastric mucosa. Other pitfalls included mucinous neoplasm vs. pancreatic intraepithelial neoplasia, endocrine tumor vs. acinar carcinoma, solidpseudopapillary neoplasm, and nesidioblastosis in chronic pancreatitis.

\begin{tabular}{|c|c|c|c|c|}
\hline Cytologic Diagnosis (n) & \multicolumn{4}{|c|}{ Histological Diagnosis } \\
\hline & Number of Case & Benign & Neoplasm & Malignancy \\
\hline Unsatisfactory (52) & 18 & 7 & 7 & 4 \\
\hline Benign (224) & 98 & 51 & 36 & {$[11$} \\
\hline Atypical (129) & 59 & 19 & 23 & 17 \\
\hline Neoplasm (140) & 91 & 8 & 74 & 9 \\
\hline Suspicious (35) & 16 & 1 & 1 & 14 \\
\hline Malignancy (632) & 114 & 2 & 1 & $\sqrt{111}$ \\
\hline
\end{tabular}

Conclusions: EUS-FNA is a valuable tool for the diagnosis of pancreatic lesions, especially solid malignant tumors. Cytologic-radiological correlation is essential in differentiating pancreatic mucinous neoplasms from gastric mucosa, since the former generally have characteristic radiological features. Pathologists should be aware of the pitfalls in cytologic diagnosis of pancreatic lesions which may significantly alter clinical management of the patients.

1839 Loss of COUP-TFII Expression Is Associated with Carcinogenesis of Pancreatic Ductal Adenocarcinoma

Z Cai, A Awadallah, WXin. University Hospitals Case Medical Center, Cleveland, OH. Background: Normal pancreatic ducts can transform into pancreatic ductal adenocarcinoma (PDAC) through pancreatic intraepithelial neoplasms (PanINs), which involves alteration of gene expression profile. Chicken ovalbumin upstream promoter-transcription factor II (COUP-TFII), also known as NR2F2, is a member of the nuclear receptor superfamily that has recently been implicated in carcinogenesis 
of breast, colorectal and prostate cancer. Here we investigate the expression pattern of COUP-TFII in normal pancreatic duct, PanINs and PDAC, aiming to define the roles of COUP-TFII in pancreatic carcinogenesis.

Design: 42 consecutive cases of primary PDAC (mean age $57 \mathrm{yrs}, \mathrm{M} / \mathrm{F}$ ratio 25/17) were selected, with matching non-neoplastic pancreatic tissues and accompanied 29 PanINs. Immunohistochemical study was performed on formalin-fixed paraffin-embedded tissue blocks using monoclonal antibody anti-NR2F2 (AbCam, Cambridge, MA). The immunostains were scored as negative (-), weakly positive $(1+)$ and strongly positive $(2+)$, as compared to the surrounding stroma.

Results: Majority of PDACs (33 of 42, 79\%) showed no expression of COUP-TFII, in contrast to normal pancreatic ducts that predominantly stained strongly positive (41 of 42, 98\%). The 10 PanIN-1 and 7 PanIN-2 lesions also readily expressed COUP-TFII while demonstrating a tendency of decreasing expression as compared to normal ducts. Significant loss of COUP-TFII expression is observed in PanIN-3 lesions, with 7 of 12 cases $(58 \%)$ showing no COUP-TFII expression, 4 (33\%) being weakly positive and 1 $(8 \%)$ retaining strongly positive.

Table 1 Immunoprofile of COUP-TFII in Normal Pancreatic Ducts, PanINs and PDACs

\begin{tabular}{|c|c|c|c|c|c|}
\hline & Normal Duct & PanIN-1 & PanIN-2 & PanIN-3 & PDAC \\
\hline Negative (-) & 0 & 0 & 0 & $7(58 \%)$ & $33(79 \%)$ \\
\hline Weakly Positive (1+) & $1(2 \%)$ & $3(30 \%)$ & $4(57 \%)$ & $4(33 \%)$ & $6(14 \%)$ \\
\hline Strongly Positive $(2+)$ & $41(98 \%)$ & $7(70 \%)$ & $3(43 \%)$ & $1(9 \%)$ & $3(7 \%)$ \\
\hline Total & 42 & 10 & 7 & 12 & 42 \\
\hline
\end{tabular}

Conclusions: Our results showed that expression of COUP-TFII sequentially decreases as normal pancreatic duct transform through PanINs into PDAC, with loss of COUP-TFII expression being a common feature of PDAC. This suggests that loss of COUP-TFII is associated with pancreatic carcinogenesis. Further study is warranted to investigate the molecular pathway(s) through which COUP-TFII is involved in pancreatic carcinogenesis.

1840 Pancreatic Endocrine Neoplasms: Is Grading Using a Ki-67 Proliferation Index Feasible and Accurate on Cytological Material

SM Chai, S Pirasteh, C Thomas, I Yusoff, P Kumarasinghe, B de Boer. PathWest Laboratory Medicine, Perth, Western Australia, Australia; Queen Elizabeth II Medical Centre, Perth, Western Australia, Australia; University of Western Australia, Perth, Western Australia, Australia.

Background: Diagnosis of pancreatic endocrine neoplasm (PEN) on fine needle aspiration (FNA) cytology is routine although the assessment of the Ki-67 proliferation index on these specimens is not. Some data suggests that a raised $(>2 \%)$ proliferation index assessed using Ki-67 correlates with more aggressive behaviour. The current WHO guidelines recommend a mitotic count in "at least 50HPFs" and Ki-67 index as a percentage of 500-2000 cells but because this is a cumbersome and time consuming process, many pathologists choose to "eyeball" the Ki-67 proliferative index despite it being shown to be much less accurate than automated counting. The current study was performed to; 1) assess the feasibility of Ki-67 scoring on cell block sections and 2) compare those results with those obtained from the corresponding histology sections. Design: Twenty patients with a diagnosis of PEN and both an adequate EUS FNA cell block and resection histopathology were selected. Both specimens were assessed for the Ki-67 proliferation index using; 1) "eyeball" examination, 2) manual counting of up to 2000 cells and 3) digital image analysis. The scores were graded using the published cutoffs: G1 $\leq 2 \%$, G2 $3-20 \%$, G3 $>20 \%$. For comparison between cytology and histopathology, a consensus grade representing a majority of the three modalities in both types of sample was used.

Results: There was agreement in grading between "eyeball" estimation, manual count and software count in 16 of 20 cell blocks and 19 of 20 histology sections. Comparison of grading between cell blocks and histology showed agreement in 15 of 20 cases and discrepant results in 5 cases, where the histology grading was one level higher than the cell block grading (four were G2 vs G1 and one was G3 vs G2). There were a number of quality control issues affecting the $\mathrm{Ki}-67$ proliferation index assessment, particularly in the cell blocks.

Conclusions: Scoring of a Ki-67 proliferation index on cell block section is feasible. There does not seem to be significant variation between "eyeball" examination, manual counting and computerized image analysis. There is reasonable correlation between cell block and histopathology grading although there is a tendency for the cell block to under call the grade, possibly explained by the lack of hot spot identification.

1841 Proposal for a Revised N-Stage for Pancreatic Ductal Adenocarcinoma as N1 $(<3)$ and N2 $(\geq 3)$ with Strong Prognostic Correlation H Choi, B Saka, S Balci, O Basturk, S Maithel, D Kooby, J Sarmiento, B El-Rayes, J Knight, $M$ Goodman, L Ducato, A Goldblum, V Adsay. Emory, Atlanta, GA; WSU, Detroit, MI.

Background: There are very few reliable prognosticators for pancreatic ductal adenocarcinoma (PDAC). Although variable data exists, lymph node (LN) involvement from PDAC is associated with poor survival. The discrepancy among different studies exists partly because surgical retrieval and pathologic assessmet of LNs has been highly variable and often inadequate, as highlighted in the NCI's SEER database showing the median number of LNs retrieved to be only "7". Current N-staging of AJCC/UICC TNM recognizes only N0 and N1 categories, but the prognostic impact of the number of metastatic LNs is not known.

Design: 250 pancreaticoduodenectomies with PDAC that had undergone "orange peeling" approach of LN harvesting were analyzed. All the LNs were re-evaluated by the authors for the presence of carcinoma as well as the number of LNs involved. Applicability and prognostic value of a revised $\mathrm{N}$-stage protocol similar to that used in other organs as N0, N1 (1-2 LNs) and, N2 ( $\geq 3)$ was analyzed.
Results: The median number of lymph nodes examined was 19(3-45). 194 (78\%) cases had LN positivity. Median number of positive LNs was $3.4(0-20)$. Median survival of $\mathrm{LN}$ negative cases was significantly better than $\mathrm{LN}$ positive cases ( $24 \mathrm{vs} 13 \mathrm{mos}$ ) $(\mathrm{p}=0.0079)$. When LN-positive cases were reclassified according to the proposed sub-staging protocol, 69 cases $(28 \%)$ were N1, and 125 cases $(50 \%)$ were N2. The median estimated survival under this new classification was 18,16 and 12 months, respectively. The 1-, 3- and 5-yr survival rates of N0 cases were $72 \%, 41 \%, 32 \%$, N1 was $66 \%, 30 \%, 16 \%$, and $\mathrm{N} 2$ was $59 \%, 19 \%, 10 \%(\mathrm{p}=0.0126)$ (Figure 1$)$. As expected, these differences seemed to be even stronger when only cases with more than $12 \mathrm{LNs}$ were analyzed separately $(\mathrm{p}=0.0104)$.

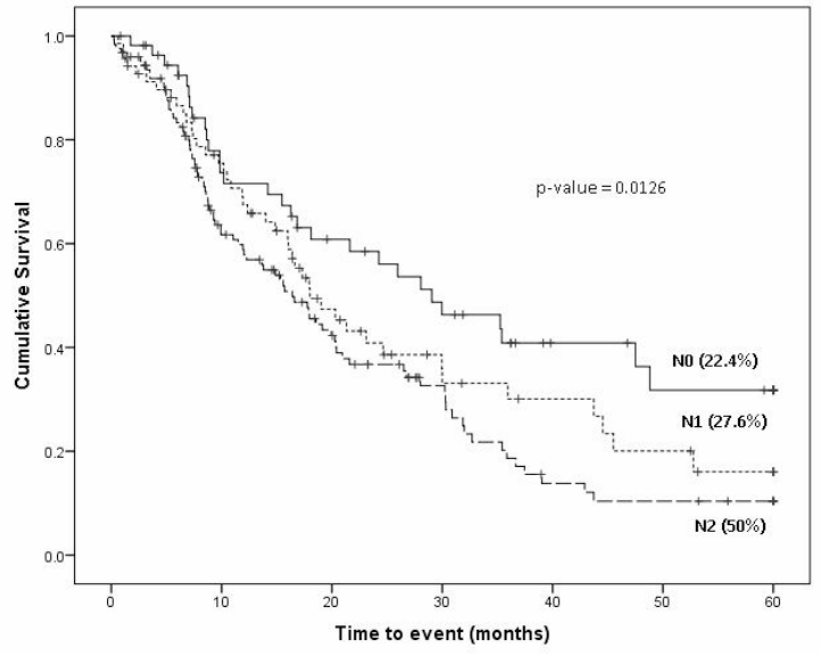

Conclusions: Sub-staging of nodal status in pancreatic ductal carcinomas as N1 and $\mathrm{N} 2$ based on the number of metastatic $\mathrm{LN}$ as $<3 \mathrm{vs} \geq 3$ has significant prognostic value. A revised N-staging protocol should be incorporated into the TNM-staging of pancreas cancers in the next edition of AJCC/UICC.

1842 Aberrant Expression of Nuclear Factor of Activated T Cells 3 (NFAT3) in Human Pancreatic Adenocarcinoma Promotes Tumor Cell Proliferation by Regulating Exression of Cell Cycle Regulating Proteins $X$ Ding, H Zhu. Saint Louis University School of Medicine, Saint Louis, MO; Mount Sinai School of Medicine, New York, NY.

Background: The NFAT family as a regulator of $\mathrm{T}$ cell activation is composed of four calcium-responsive isoforms. Recent studies suggest that NFAT1 and NFAT2 are key regulators of neoplastic transformation and cancer progression but the role of NFAT3 is still unknown. This project aims to study expression of NFAT3 in human pancreatic adenocarcinoma and decipher the impact of NFAT3 on pancreatic cancer cell proliferation.

Design: Immunostaining for NFAT3 was performed on 18 paraffin-embedded human pancreatic adenocarcinoma, their adjacent PanIN lesions and paired non-neoplastic pancreas. The proper positive and negative controls were also established. Based on the staining intensity, the staining of the pancreatic tissues was classified as no staining $(0)$, low intensity staining $(+)$ or high intensity staining $(++)$. We use two human pancreatic cancer cell lines (AsPC-1 and PANC-1) to decipher the effect of NFAT3 on cancer cell proliferation in vitro. Small interfering RNA (siRNA) and cyclosporine A were used to block NFAT3 expression and activity. Flow cytometry was used for cell cycle analysis. Expression of cell cycle regulating proteins was evaluated by western blot.

Results: Distinct aberrant expression of NFAT3 was identified in infiltrating pancreatic ductal adenocarcinoma. 78\% (14/18) pancreatic adenocarcinoma show intense staining for NFAT3. The positive staining was identified in cytoplasm and nuclei of tumor cells. NFAT3 was negative in normal pancreas, chronic pancreatitis and all PanIN lesions. Inhibition of NFAT3 by siRNA or cyclosporine A markedly blocks pancreatic cancer cell proliferation and arrests tumor cells at G0/G1 phase in vitro. Western blot analysis reveals that inhibition of NFAT3 significantly decreases expression of two cell cycle regulating proteins, Cyclin D1 and Cyclin E.

Conclusions: This study has clearly demonstrated an aberrant expression of NFAT3 in human infiltrating pancreatic adenocarcinoma. NFAT3 might be a useful malignant biomarker to differentiate pancreatic adenocarcinoma from pre-malignant and nonneoplastic lesions. NFAT3 regulates pancreatic cancer cell proliferation through down-regulating Cyclin D1 and Cyclin E expression, which further warrants exploring the role of NFAT in pancreatic carcinogenesis and as a potential target for treatment of pancreatic cancer.

1843 Solid Pseudopapillary Neoplasm of the Pancreas: Clinicopathologic and Survival Analyses of 64 Cases from a Single Institution

J Estrella, L Li, A Rashid, H Wang, MH Katz, JB Fleming, JL Abbruzzese, H Wang. UT MD Anderson Cancer Center, Houston, TX; Changhai Hospital, The Second Military Medical University, Shanghai, China.

Background: Although solid pseudopapillary neoplasms (SPNs) are considered tumors of low malignant potential, patients may present with aggressive disease (i.e. liver 
metastasis/invasion into adjacent organs), and rarely, die from their disease. While the clinicopathologic features associated with aggressive SPNs have been reported, important prognostic factors of survival remain unclear.

Design: We systematically reviewed 64 cases of SPN resected at our institution for tumor size, extent of invasion, margin status, presence of lymphovascular invasion, muscular vessel invasion, perineural invasion, lymph node and/or distant metastases. Clinicopathologic characteristics were correlated with the presence of metastasis/ recurrence and disease-specific survival.

Results: Five (8\%) patients presented with stage IV disease. Among them, 2 died of disease (one at 2.5 months due to extensive peritoneal tumor involvement and one at 19 months due to multiple liver metastasis and peritoneal seeding), 2 were alive with disease at 69 and 86 months and one had no evidence of disease at 44 months after resection of recurrent disease. During follow-up, 5 (13\%) of 39 patients with stage I-II disease had recurrences. Patients with metastatic/recurrent SPNs had significantly larger tumor size $(\mathrm{p}<0.001)$ and more frequent tumor invasion of muscular vessels $(\mathrm{p}=0.02)$. Tumor invasion into muscular vessels $(\mathrm{p}=0.001)$, Tumor $(\mathrm{T})$ stage by European Neuroendocrine Tumors Society (ENETS) classification $(\mathrm{p}<0.001)$, ENETS stage grouping $(\mathrm{p}<0.001)$ and stage grouping by the American Joint Committee on Cancer (AJCC stage, $p<0.001$ ) are important predictors of disease-specific survival in patients with SPN.
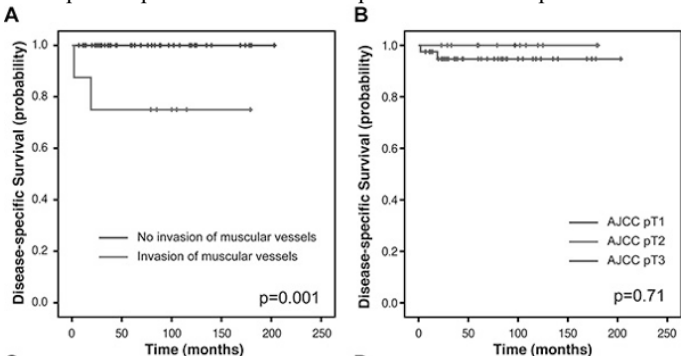

C
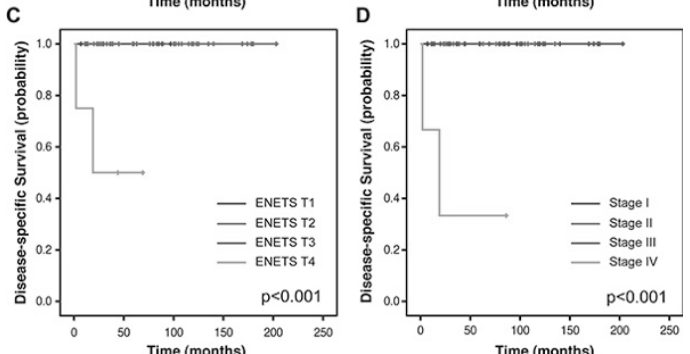

Conclusions: Our study highlights the importance of including the presence of vascular invasion and staging information in surgical pathology reports, for risk assessment and guide clinical management in patients with SPNs.

1844 GNAS Mutations Are Associated with an Intestinal Lineage IPMN L Fazlollahi, D Dias-Santagata, J Solus, LA Bernardo, G Marchegiani, C Fernandez-del Castillo, MB Pitman, M Mino-Kenudson. Massachusetts General Hospital, Boston, MA. Background: Intraductal papillary mucinous neoplasms (IPMN) are classified into 4 epithelial types intestinal [IPMN-I], gastric [IPMN-G], pancreatobiliary [IPMN-PB], and oncocytic [IPMN-O], each one is associated with unique clinicopathological features and biology. Of those, IPMN-I makes up for the majority of main duct-type IPMN often exhibits high-grade dysplasia (HGD) with or without invasive carcinoma. KRAS and $G N A S$ mutations are found in $60-70 \%$ of IPMNs. Interestingly GNAS mutations have been reported in villous adenomas of the colorectum and low-grade appendiceal mucinous neoplasms that exhibit similar morphology to IPMN-I. Thus, we hypothesized that $G N A S$ mutations are associated with an intestinal lineage in IPMN.

Design: We analyzed GNAS and KRAS mutations in $60 \mathrm{IPMN}$ foci with various epithelial types and dysplastic grades from 44 patients using a PCR-based multiplex assay. Results: $G N A S+/-K R A S$ mutations were identified in $>90 \%$ of IPMN-I, irrespective of grade. In IPMN-G, $65 \%$ of foci harbored $G N A S+/-K R A S$ mutations and $24 \%$ exhibited $K R A S$ mutations only. However, $90 \%$ of IPMN-G foci found in association with IPMN-I $(\mathrm{n}=10)$ showed $G N A S+/-K R A S$ mutations. Although limited in number, IPMN-PB with or without microinvasion often exhibited $K R A S$ mutations only. Table 1

\begin{tabular}{|c|c|c|c|c|}
\hline & $\begin{array}{l}\text { KRAS mut// } \\
\text { GNAS wt }\end{array}$ & $\begin{array}{l}\text { GNAS mut// } \\
\text { KRAS wt }\end{array}$ & $\begin{array}{l}\text { KRAS mut// } \\
\text { GNAS mut }\end{array}$ & $\begin{array}{l}\text { KRAS wt/GNAS } \\
\text { wt }\end{array}$ \\
\hline Intestinal (\%) & $0(0)$ & $7(41)$ & $9(53)$ & $1(5.9)$ \\
\hline IGD (\%) & $0(0)$ & $6(43)$ & $7(50)$ & $1(7)$ \\
\hline HGD (\%) & $0(0)$ & $1(33)$ & $2(67)$ & $0(0)$ \\
\hline Gastric (\%) & $9(24)$ & $5(14)$ & $19(51)$ & $0(0)$ \\
\hline LGD* (\%) & $4(21)$ & $3(16)$ & $9(47)$ & $3(16)$ \\
\hline IGD (\%) & $5(29)$ & $2(12)$ & $9(53)$ & $1(5.9)$ \\
\hline HGD (\%) & $0(0)$ & $0(0)$ & $1(100)$ & $0(0)$ \\
\hline Pancreatobiliary (\%) & $2(67)$ & $0(0)$ & $0(0)$ & $1(33)$ \\
\hline Oncocytic (\%) & $0(0)$ & $0(0)$ & $0(0)$ & $1(100)$ \\
\hline $\begin{array}{l}\text { Microlnvasive PB** } \\
(\%)\end{array}$ & $1(100)$ & $0(0)$ & $0(0)$ & $0(0)$ \\
\hline $\begin{array}{l}\text { MicroInvasive Intestinal } \\
* *(\%)\end{array}$ & $0(0)$ & $0(0)$ & || $1(100)$ & $0(0)$ \\
\hline
\end{tabular}

* LGD: low-grade dysplasia **microinvasive carcinoma arising in IPMN-PB or IPMN-I
Among the 9 cases with multiple foci examined, 5 cases with IPMN-I and IPMN-G foci and 1 case with different grades of IPMN-I]harbored exact same mutations in all foci within one case. Conversely, 3 cases contained IPMN-PB or IPMN-O showed different $K R A S$ mutations in high-grade foci and IPMN-G, suggesting multi-lineage lesions.

Conclusions: The vast majority of IPMN-I and IPMN-G foci in the background of IPMN-I harbor GNAS mutations, suggesting GNAS mutations are involved in intestinal differentiation in IPMN. Our data also suggest some IPMN lesions, especially with pancreatobiliary- and/or oncocytic-type epithelium, molecularly appear to be multilineage. A larger cohort study to confirm these findings is underway.

1845 AST/GOT1 Expression Status in Primary Resection Specimen Is a Prognostic Biomarker in Human Pancreatic Ductal Adenocarcinoma FM Feld, PD Nagel, C Welke, P Moller, JK Lennerz. University Ulm, Ulm, Baden Wuerttemberg, Germany.

Background: Oncogenic $K R A S$ mutations are a hallmark of pancreatic ductal adenocarcinoma. Recently, a metabolic function of the KRAS mutation has been established (nature 2013; Apr 4; 496:101-5). Specifically, KRAS acts as a modulator of the cancer cell's redox maintenance system, which goes along with an increase in the glutamic-oxalacetic transaminase 1 (GOT1) expression. The clinical relevance of this metabolic KRAS-GOT link has not yet been assessed in primary human patient samples. Thus, here we studied whether the GOT1 expression status (a) correlates with the KRAS mutation status, (b) can be identified via specific clinicopathological phenotypes and (c) has prognostic relevance.

Design: Two independent cohorts were analyzed: the first, 'international cancer genome consortium; (ICGC, 57 patients with whole-exome sequencing data, genome wide expression profile, clinicopathological (CPC) data and follow up) and second, 'Ulm' (124 patients with basic molecular genetic workup, tissue-microarray, CPC data and follow up). GOT1 expression was assessed via RNA levels (ICGC) and by immunohistochemistry (Ulm). Contingency and outcome analyses employed t-tests, Fisher's exact test, chi-square and log-rank statistics. Multivariate comparisons were modeled after univariate results; statistical significance was defined as $P<0.05$.

Results: The GOT1 expression status was not predictive of the KRAS mutation status ( $P=0.2$ (ICGC), $P=0.7$ (Ulm); both Chi-square). The lack of association indicates that GOT1 assessment cannot serve as a surrogate marker for KRAS mutation testing and that additional modulatory factors are at play in the $K R A S$-GOT axis in primary human tumor samples. Phenotype comparison revealed that GOT1 high/positive subgroups do not differ by age, sex, T, N, M, overall stage grouping or tumor grading ( $P=0.39$ 1.00 ). Thus, the GOT1 subgroups cannot be distinguished based on clinicopathological phenotype. Outcome analysis revealed that patients with GOT1 high-expressing tumors had significantly longer overall survival times when compared to the low-expressing tumors ( $P=0.0483$ ICGC), which was verified by immunohistochemistry in the Ulm cohort $(P=0.0488)$. Uni- and multivariate analyses and cohort comparisons showed that GOT1 expression status is an independent prognostic factor in up to $68 \%$ of tumors. Conclusions: The biology of the KRAS-GOT mechanism is seemingly more complex than in cellular models and will have to be delineated; however, our results specify that the GOT1 status is a valuable prognostic biomarker in pancreatic ductal adenocarcinoma.

1846 The Pathology of Type 2 Autoimmune Pancreatitis: A Retrospective Analysis of Cases Resected over Last 2 Decades

RK Gupta, V Deshpande. University of Tennessee HSC - College of Medicine, Memphis, TN; Massachusetts General Hospital and Harvard Medical School, Boston, MA Background: The 2 variants of autoimmune pancreatitis (AIP), type 1 AIP and type 2 AIP are associated with distinct clinical and histopathologic features. Although the features of type 2 AIP have been delineated in previous studies, there has been no systematic evaluation of the pathology of type 2 AIP in North America.

Design: Pancreatic resections between 1993 and 2012 diagnosed as chronic pancreatitis at Massachusetts General Hospital were reviewed. The available H\&E slides were graded for the intensity of chronic periductal infiltrate from 0 to 3 . Active inflammation in the form of neutrophils were also graded from mild, moderate (abscesses) and severe (ulceration). A diagnosis of type 2 autoimmune pancreatitis required the presence of either grade 2 periductal chronic inflammation or grade 1 associated with neutrophils. Relevant demographic and clinical data was collected. Immunohistochemistry for IgG4 was performed.

Results: We identified 38 cases of type 2 AIP, constituting $17.3 \%$ of cases analyzed. The cohort consisted of 34 Whipple resections and 4 distal pancreatectomy specimens. The mean age of the patients was 51 years. A slight male predominance was noted $(\mathrm{M}: \mathrm{F}=21: 17)$. The most common presenting symptom was abdominal pain ( 33 cases) followed by weight loss (15 cases) and jaundice ( 6 cases). History of alcohol abuse and smoking were identified in 5 cases each. A diagnosis of pancreatitis (14 cases) or pancreatic mass ( 12 cases) was the usual indication for pancreatic resections. The periductal lymphoplasmacytic infiltrate ranged between grade 1 ( 9 cases), grade 2 (15 cases) and grade 3 (14 cases).The intensity of neutrophilic infiltrate also varied considerably. Intraductal abscesses with tissue necrosis were seen in 4 cases and acinar inflammation in 15 cases. Dense interlobular fibrosis was identified in 34 cases. Other findings included non-obliterative phlebitis ( 9 cases), prominent eosinophilia (10 cases), lymphoid aggregates ( 7 cases) and periductal granulomas ( 2 cases). Six patients had a history of inflammatory bowel disease. 5 out of 8 cases showed less than $10 \operatorname{IgG} 4$ positive cells per HPF.

Conclusions: The presence of periductal infiltrate defines a unique variant of pancreatitis. In this surgical series type 2 autoimmune pancreatitis is a common inflammatory disease of the pancreas. Some cases of type 2 AIP however shows overlapping clinical and histological features with ethanol-related chronic pancreatitis. 
1847 Prognostic Role of Neuroendocrine Differentiation in Pancreatic Ductal Adenocarcinoma: A Retrospective Single-Center Study

RK Gupta, DD Santos, C Ferrone, V Deshpande. University of Tennessee HSC College of Medicine, Memphis, TN; Massachusetts General Hospital, Boston, MA.

Background: Foci of neuroendocrine differentiation are identified in virtually all solid epithelial tumors of the gastrointestinal tract, including pancreatic adenocarcinoma (PDAC). Previous studies have suggested that the presence of neuroendocrine cell differentiation in pancreatic adenocarcinoma predicts improved survival. We investigate the prognostic relevance of neuroendocrine cell differentiation in a large cohort of pancreatic adenocarcinomas resected with the intent to cure.

Design: We identified a cohort of 391 PDACs with available follow-up information. Tissue microarrays (TMAs) composed of $3 \mathrm{~mm}$ core of tissue were constructed from these specimens. At least 2 cores were included from each patient. Clinical, demographic and survival data were recorded. An immunohistochemical stain for chromogranin was performed and the reactivity of the tumor cells was scored as positive or negative. Reactivity within tumor cells was distinguished from that in neuroendocrine cells colonizing malignant glands which was also scored similarly. This latter phenomenon was manifest by the presence of neuroendocrine cells at the abluminal surface of the ducts.

Results: Chromogranin reactivity was identified in 32 of 391 cases $(8.1 \%)$. The reactivity in tumor cells varied from rare weakly positive staining to diffuse and strong staining. The immunoreactivity within the neoplastic cells was significantly less than in normal islets and endocrine cells involved in the colonization. Although the PDACs with positive staining showed a lower median survival when compared to those without reactivity for chromogranin (estimated median survival 42 months versus 49 months), this difference was not statistically significant (log rank test 0.8$)$. Colonization was identified in 109 cases $(28 \%)$. There was no difference in the median survival between cases with colonization (48 months) and those without colonization (49 months) (log rank test 0.9 ).

Conclusions: The presence of neuroendocrine reactivity within pancreatic ductal adenocarcinoma does not influence overall survival. Colonization by neuroendocrine cells is common in PDAC, and represents a potential pitfall, particularly on biopsy samples.

\section{GNAS Mutations Are Frequent in Pancreatic Adenocarcinomas} with Mucinous Phenotype

W Hosoda, E Sasaki, Y Murakami, Y Yatabe. Aichi Cancer Center Hospital, Nagoya, Japan.

Background: GNAS encodes the alpha-stimulatory subunit of heterotrimeric G-proteins which is crucial for the cyclic AMP-mediated signal transduction. Recently, intraductal papillary mucinous neoplasm (IPMN) has been reported to show frequent GNAS mutations, in contrast to pancreatic ductal adenocarcinoma (PDA). The aim of this study is to examine clinicopathological characteristics of GNAS mutation in various pancreatic carcinomas.

Design: We examined codon 201 of GNAS and codon 12, 13, and 61 of KRAS using a series of 290 surgically resected pancreatic tumors, including 88 PDAs, 3 colloid carcinomas, 29 IPMN-associated adenocarcinomas, 61 IPMNs, 10 mucinous cystic neoplasms, 52 neuroendocrine neoplasms, 16 acinar cell neoplasms, 10 serous neoplasms, and 14 solid-pseudopapillary neoplasms, in addition to microdissected 77 PanIN lesions. In 29 IPMN-associated adenocarcinomas, the components of IPMN and adenocarcinoma were microdissected and examined separately.

Results: GNAS mutations were frequently detected in colloid carcinomas $(67 \%, 2 / 3)$, IPMNs $(64 \%, 39 / 61)$, and IPMN-associated adenocarcinomas ( $38 \%, 11 / 29)$. In contrast, other histological subtypes including PDAs $(1 \%, 1 / 88)$ and a precursor lesion of PDA (PanIN, 3\%, 2/77) rarely harbored GNAS mutation. Frequent KRAS mutations were detected in PDAs $(92 \%, 81 / 88)$, IPMN-associated adenocarcinomas $(76 \%, 22 / 29)$, IPMNs $(59 \%, 36 / 61)$, and mucinous cystic neoplasms $(40 \% .4 / 10)$. When IPMNassociated adenocarcinomas were subcategorized into mucinous and tubular subtypes, the mucinous subtype showed higher frequency of GNAS mutation $(83 \%, 5 / 6)$ than those of the latter subtype $(26 \%, 6 / 23)(\mathrm{p}=0.02)$. Furthermore, mucinous subtypes shared clinicopathological characteristics with IPMN. The difference between the two subtypes in IPMN-associated adenocarcinoma were highlighted by concordance of the genotypes between the components of IPMN and adenocarcinoma; all mucinous-subtype tumors showed identical genotypes between the two components in individual tumors, whereas 8 of 23 tubular subtypes were different in their genotypes.

Conclusions: Our study suggested that GNAS mutated tumors, including IPMN, IPMNassociated adenocarcinoma, particularly mucinous subtype, and colloid carcinoma, constituted a spectrum of pancreatic tumors. Although the clinicopathological association among these tumors have been reported, GNAS mutation further sheds light on the spectrum

\section{ZIC2 Is an Indispensable Transcriptional Factor for the Cell Growth} of Pancreatic Ductal Adenocarcinoma

S Inaguma, K Kasai, M Hashimoto, M Riku, H Ikeda. Aichi Medical University School of Medicine, Nagakute, Aichi, Japan.

Background: ZIC2 is one of five members of ZIC family gene that contain a C2H2-type of zinc fingers and function as a transcriptional regulator. As a part of Hedgehog signal molecules, ZIC2 is indispensable for the development of central nervous system and its mutation causes holoprosencephaly, the most common structural anomaly of the human brain. Recently, we uncovered that ZIC2 is a unique member which is over-expressed in all (11/11) of the pancreatic ductal adenocarcinoma (PDAC) cell lines we tested. We also found that ZIC2 knockdown by the siRNAs lead to the apoptotic cell death of PDAC cells. In contrast, Tet-induced ZIC2 expression accelerated PDAC cell proliferation. In this session, we would like to highlight the importance of ZIC2 over-expression in PDAC cells from the point of ZIC2 transcriptional target genes.

Design: We employed siRNA-mediated ZIC2-knockdown PANC-1 cells and Tetinduced ZIC2-over-expressed PANC-1 cells for cDNA expression microarray analysis using Agilent $4 \mathrm{x} 44 \mathrm{~K}$ cDNA microarray. Concerning the identified ZIC2 transcriptional targets, we performed over-expression and knockdown experiments using PDAC cells to uncover how the ZIC2 transcriptional targets regulate PDAC cell apoptosis and growth. Finally, we performed immunohistochemical analysis to assess the relationship between the ZIC2 and its target gene expressions or cell proliferation marker Ki-67 labeling index in 20 cases of resected PDAC tissue.

Results: Among the 43 identified ZIC2 target genes, we focused on ANXA8L2 and FGFR3 in this session. Forced expression of ANXA8L2 partly reduced the apoptotic cell death of the ZIC2-knockdown PDAC cells. On the other hand, combined transfection of the ZIC2 expression vector and FGFR3 specific siRNAs revealed that ZIC2 activated RAS-MAPK pathway in a FGFR3 dependent manner. The ZIC2, ANXA8L2 and FGFR3 expressions were immunohistochemically under-detectable levels in the normal pancreatic duct. Their expression was weakly identified in low-grade PanIN and prominent in high-grade PanIN and PDAC cells, respectively. There were positive correlation between ZIC2 and ANXA8L2 $(\rho=0.774, \mathrm{p}<0.0001)$ or FGFR3 $(\rho=0.828$, $\mathrm{p}<0.0001)$ expression. We also uncovered the positive correlation between the ZIC2 expression and Ki-67 labeling index $(\rho=0.773, p<0.0001)$.

Conclusions: Our experimental results indicate that ZIC2-ANXA8L2 and ZIC2FGFR3-MAPK axis are important signaling pathways for the regulation of apoptosis and proliferation in the PDAC development.

\section{Mucinous Carcinomas of the Ampulla: Clinicopathologic Analysis} of 33 Cases

K-T Jang, S Balci, P Bagci, B Saka, N Dursun, O Basturk, A Krasinskas, G Kim, N Ohike, T Tajiri, J Knight, VAdsay. SMC, Seoul, Korea; Emory, Atlanta, GA; MSKCC, NY, NY; UCSF, SF, CA; Showa, Tokyo, Japan; Tokai, Tokyo, Japan.

Background: There is virtually no information on the frequency and clinicopathologic characteristics of ampullary mucinous carcinomas(MCs).

Design: 359 well-characterized ampullary carcinomas (AC) were reviewed. Nonampullary duodenal, pancreatic \& distal CBD carcinomas were excluded by purist's approach.

Results: Mucinous differentiation (stromal mucin deposition) was identified in 59 ACs (16\%). $26(7 \%)$ of these had mucin $<50 \%$ of the tumor and were excluded as carcinoma with focal mucin production, and the remaining $33(9 \%)$ was further analyzed as MC and contrasted with other $\mathrm{ACs}: \mathrm{M} / \mathrm{F}=24 / 8$ ( $v s$ 182/138); mean age $=63$ (vs 65 ). 27\% of MCs were from the duodenal surface of the ampulla ( $v s 5 \%$ ). Invasive carcinoma size was larger for MCs (mean, 25.3 vs $18.1 \mathrm{~mm}$; $\mathrm{p}=0.001$ ). 30\% contained acellular mucin lakes, but only $6 \%$ were pure colloid type. Signet ring cells in mucin were identified in $36 \%$. Non-mucinous component (cellular infiltrates into the stroma) was present in 28/33 and often contained multiple patterns in a mixture: Tubular-intestinal 13 , tubular-pancreatobiliary 12 , micropapillary 7 , poorly diff 7 , signet ring cell 6 , and squamous 1 . Almost half (46\%) had significant preinvasive (adenomatous) component, mostly intestinal $(65 \%)$ or mixed $(22 \%)$; rarely gastro-pancreatobiliary lineage $(11 \%)$. None of the 15 MCs analyzed revealed loss of MMR proteins by IHC ( $v s$ loss in 12/68 other ACs and in 6/11 carcinomas with focal mucin). T stage distribution was similar to other ACs. The frequency of LN metastasis was higher but this was not statistically significant ( $63 \%$ vs $44 \%$; $\mathrm{p}=0.06)$. Overall 3- \& 5-yr survival was not different from other ACs $(59 \%$ and $49 \%$ vs $61 \%$ and $47 \%$; $=0.92)$

Kaplan-Meier Survival Curves

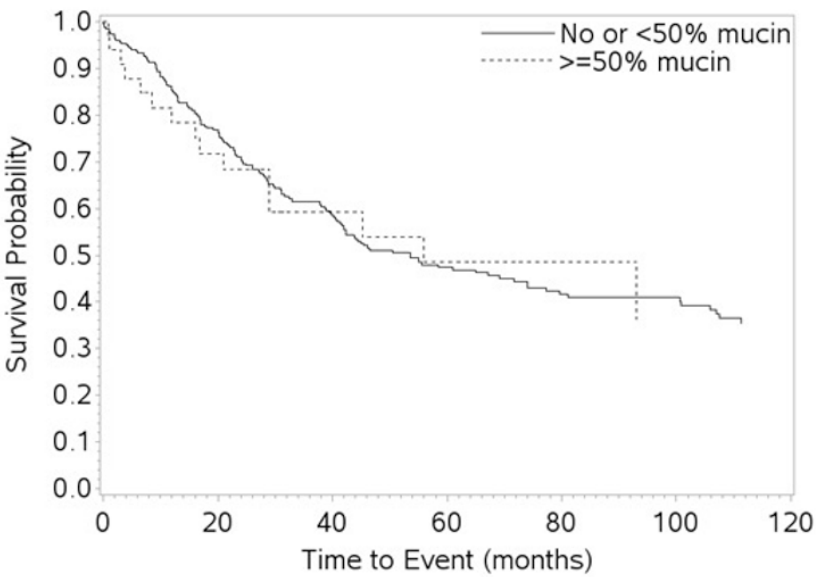

Of note, including carcinomas with focal mucin did not bring any survival difference. Conclusions: $\mathrm{MC}$ (stromal mucin $>50 \%$ of the tumor) constitutes $9 \%$ of ACs. Most are mixed-mucinous type. Almost half are associated with adenomatous component, mostly intestinal type. Unlike their lower-GI counterparts, these tumors do not show association with MSI, although those with lesser mucin production might. Despite their large size and common (63\%) LN metastasis, the prognosis of these tumors does not seem to be worse than that of other ACs. 
1851 P53 Alterations in Acinar Cell Carcinomas of the Pancreas: New Insights into the Pathogenesis of Such Rare Cancers

$S$ La Rosa, B Bernasconi, M Frattini, N Sahnane, F Molinari, MG Tibiletti, D Furlan, L Zang, A Vanoli, S Casnedi, V Adsay, K Notohara, L Albarello, S Asioli, F Sessa, C Capella. Ospedale di Circolo, Varese, Italy; University of Insubria, Varese, Italy; Institute of Pathology, Locarno, Switzerland; Mayo Clinic, Rochester, MN; University of Pavia, Pavia, Italy; Hospital De Hautepierre, Strasbourg, France; Emory University, Atlanta, GA; Kurashiki Central Hospital, Kurashiki, Japan; San Raffaele Hospital, Milan, Italy; University of Turin, Turin, Italy.

Background: The role of $\mathrm{p} 53$ in the pathogenesis of pancreatic acinar cell carcinomas (ACCs) is not clear. A few studies, mainly using immunohistochemistry, suggested that p53 is not altered in ACCs, but the small number of cases considered and the molecular analyses performed did not provide definitive data. Moreover, p53 mutation in $1 / 5$ ACCs (Mod Pathol 24:1229,2011) and a significant ( $>50 \%$ of cells) p53 nuclear immunoreactivity (IR) in 27\% of ACCs (Am J Surg Pathol 36:1782,2012) have been recently demonstrated. For these reasons, the role of $\mathrm{p} 53$ in the pathogenesis of ACCs still needs further investigation.

Design: We investigate p53 alterations (mutation, methylation, and loss) in 44 ACCs using direct sequencing of exons 4-8, MS-MLPA, and FISH. In addition, we evaluated nuclear p53-IR by immunohistochemistry.

Results: p53 mutations were found in 8/44 (18\%) cases. They correlated with higher tumor stage ( 5 cases were at stage IV) and, in one case, it was observed in the metastasis of a primary p 53 wild type ACC. p53 gene loss, including $17 \mathrm{p} 13$ deletion and monosomy of chromosome 17, was found in $50 \%$ of ACCs and it was associated with mutation in 4 cases. p53 methylation was observed in only one case. p53 alterations correlated with p53-IR. The simultaneous presence of both p53 mutation and loss correlated with worse prognosis ( $\mathrm{p}: 0.001$ )

Conclusions: p53 alterations including mutations and cytogenetic loss are frequent in ACCs and correlated with higher tumor stage and a more aggressive behavior suggesting that $\mathrm{p} 53$ is not early involved in ACC tumorigenesis, but later in cancer progression.

1852 Differential Expression of miR-196a and miR-216a in Cholangiocarcinoma and Pancreatic Ductal Adenocarcinoma

$J$ Lai, BK Larson, S Thakur, SS Sahu, S Agarwal, M Guindi, K Kalra, D Dhall, RK Pillai. Cedars-Sinai Medical Center, Los Angeles, CA; BioGenex Inc, Fremont, CA. Background: Cholangiocarcinomas (CC) and pancreatic ductal adenocarcinomas (PDAC) are virtually indistinguishable histologically and have overlapping immunohistochemical profiles. MicroRNAs (miRNAs) are short non-coding RNAs involved in post-transcriptional regulation of gene expression. Dysregulation of miRNAs and their cognate targets are increasingly implicated in many cancers. Use of microRNA in diagnostic applications has been limited by inability to determine expression patterns in situ on a cellular level, as profiling-based strategies using tissue homogenates may not adequately resolve expression differences in tumor microenvironment.

Design: 13 cases of CCs (12 intrahepatic and 1 extrahepatic) and 18 cases of PDACs were used in the study. Cases were subjected to in situ hybridization (ISH) using FAMlabeled miRNA probes (BioGenex) for miR-21, miR-10b, miR-196a and miR-216a followed by super sensitive ISH Detection Kit (BioGenex, DF400-YAX). Nuclear staining was evaluated semi-quantitatively by intensity (low, no or weak stain; high, moderate to intense stain). Statistical analysis was performed by using Fisher's exact two-tailed test.

Results: High level expression of both nuclear miR-196a and miR-216a expression was seen in $85 \%$ (11/13) of CC. PDAC cases showed high level expression of nuclear miR$196 \mathrm{a}$ and miR-216a expression in $22 \%(4 / 18)$ and $28 \%$ (5/18) cases, respectively, which was significantly different from $\mathrm{CC}(\mathrm{p}<0.01)$. No significant difference in the expression of miR-21 and miR-10b was identified between the CCs and PDACs (both P $>0.05$ ). Conclusions: miR-196a and miR-216a expression were upregulated in CC compared to PDAC. This study demonstrates feasibility of an "in situ" evaluation in FFPE where morphologic control of cells of interest and differentiating cancer and benign cell expression is retained. Ongoing studies in a larger cohort are underway to fully assess the potential of these markers in differentiating CC from PDAC.

1853 Histological Subtyping of Ampullary Carcinomata - Does It Matter? JM Leo, SE Kalloger, R Peixoto, DL Webber, DA Owen, D Renouf, DF Schaeffer. Vancouver General Hospital, UBC, Vancouver, BC, Canada; BC Cancer Agency, Vancouver, BC, Canada.

Background: Ampullary carcinomata (AC) are defined as tumors that completely replace, surround or are centered on the ampulla of Vater. Histologically, they are separated into intestinal (IT) or pancreatobiliary (PB) subtypes. Although morphologic, immunohistochemical and molecular differentiation of AC-IT and AC-PB have been well documented the prognostic significance of histologic subtype is unclear. Additionally, whether patients with either subtype benefit from pancreas or intestinal specific chemotherapeutic treatment.

Design: We identified patients in a retrospective fashion who underwent either an ampullectomy or pancreaticoduodenectomy for AC at Vancouver General Hospital between 1997 and 2012. Demographic information, pathological variables and outcome/ survival information was recorded. Patients, with an R0 resection, were divided into 2 groups based on tumor morphology (pancreatobiliary: cuboidal lining; intestinal: columnar cells and/or cribriform architecture) and immunohistochemistry (AC-IT: CK7-/CK20+/CDX2+; AC-PB: CK7+/CK20-/CDX2-).

Results: The cohort consisted of 33 patients [mean age 65.5 yr [41-82]; m:f = 15:18; IT: $n=16 ;$ PB: $n=17]$. Ninety percent of patients within the cohort did not receive initial adjuvant chemotherapy, either due to patient preference, poor performance status, or low-risk clinical characteristics, yielding a 3-year progression free survival (PFS) of
AC-IT 72\% vs AC-PB 55\% ( $p=0.27$ ) and a 3-year overall survival (OS) of AC-IT 53\% vs AC-PB $46 \%(p=0.57)$ in the untreated cohort. Three patients with AC-PB were initially treated with 5 -FU $(n=2)$ and gemcitabine $(n=1)$ regimes; an additional AC-PB patient, without initial chemotherapy, had a recurrence 17 months after resection and was subsequently treated with gemcitabine. One patient initially treated with 5-FU recurred 9 months after start of therapy, while the two patients on gemcitabine were alive at 3 years. OS for the entire cohort at 3 years demonstrated no difference between the two histological subtypes with $63 \%$ of patients alive.

Conclusions: Although OS and PFS were not statistically significant in our cohort, patients with PB subtype tended to show a lower probability of survival and higher chance of recurrence at 3 years. Interestingly, two AC-PB patients treated with gemcitabine were alive three years after resection. Despite the small sample size and short follow-up this study highlights the heterogenous nature of AC and makes the case that histological subtyping may be of value to stratify patients for chemotherapeutic treatment.

\section{CD5 Is Highly Sensitive and Specific for Pancreaticobiliary}

\section{Adenocarcinoma}

GH Lewis, RB West, RK Pai, TA Longacre. Stanford University, Stanford, CA; University of Pittsburgh, Pittsburgh, PA.

Background: Determining the site of origin in cases of metastatic adenocarcinoma of unknown primary, where the differential diagnosis includes pancreatobiliary and upper gastrointestinal primaries presents a challenge for pathologists. Sensitive and specific markers for these sites are lacking. The expression of CD5 and CD7 in epithelial neoplasms has previously been reported. We studied the expression of CD5 and CD7 in a series of tissue microarrays (TM) containing pancreatobiliary (PBA), gastric (GA), breast (BrA), and lung (LA) adenocarcinomas and report the sensitivity (SN), specificity (SP), positive (PPV) and negative predictive values (NPV) for predicting site of origin. Design: TM containing $62 \mathrm{PBA}, 82 \mathrm{GA}, 19 \mathrm{BrA}$, and $8 \mathrm{LA}$ were stained for $\mathrm{CD} 5$ and CD7. Any expression was scored positive.

Results: CD5 expression was seen in $87 \%$ of PBA, $44 \%$ of GA, $16 \%$ of $\mathrm{BrC}$, and $0 \%$ of LA. CD7 was negative in all cases except $2 \%$ of GA. Compared only to $\mathrm{BrC}$ and LA, the SN, SP, PPV, and NPV for PBA are $87 \%, 89 \%, 95 \%$, and $75 \%$, respectively; while for GA they are $44 \%, 89 \%, 92 \%$, and $34 \%$, respectively. Compared to BrC, LA, and GA, the SN, SP, PPV, and NPV for PBA is $87 \%, 64 \%, 58 \%$, and $90 \%$. The SN, SP, PPV, and NPV of CD7 for GA is $2 \%, 100 \%, 100 \%$, and $53 \%$.

Conclusions: CD5 is highly sensitive and specific for PBA especially if GA is not in the differential diagnosis. While CD7 is highly specific for GA, it is not as diagnostically useful due to low sensitivity. CD5 is a potentially useful marker for the diagnosis of PBA and to a lesser extent, GA in metastatic adenocarcinoma of unknown primary.

1855 Excision Cross-Complementing Gene1 and X-Ray Repair CrossComplementing 1 Gene as Prognostic Markers in Biliary Cancer

M Mian, MG McNamara, DW Hedley, JJ Knox, S Serra. University Health Network, Toronto, Canada.

Background: Excision cross-complementing gene1 (ERCC1) and X-ray repair crosscomplementing 1 gene (XRCC1) are important for DNA repair. Previous studies have thus examined the tissue expression of these genes in a variety of cancers but the results affecting treatment outcomes have been variable. One study in biliary cancer examined ERCC1 expression but did not relate this to treatment. The aim of the current study was to evaluate the expression of both ERCC1 and XRCC1 protein in biliary tract malignancies and access the prognostic value of these markers on outcome.

Design: All cases of biliary tract (gallbladder, extrahepatic, Klatskin and intrahepatic cholangiocarcinomas) cancer with available tumour from 2000 to 2013 diagnosed at University Health Network, Toronto, Canada were initially chosen and those with adequate follow-up were selected for the study. Relevant clinicopathological information, chemotherapy and survival data were obtained. A TMA was constructed and stained for ERCC1 and XRCC1. Each case was scored as high or low based on a product of intensity and percentage of tumour cells stained.

Results: Of 71 patients, 40 were males. The mean patient age was 65.5 years with mean overall survival of 26 months (range 0.5 - 97.9 months). Twenty eight (39.4\%) patients received adjuvant chemotherapy. Immunohistochemistry showed $40(56.3 \%)$ high XCCR1 (-H) and 18 (25.4\%) high ERCC1 (-H) tumors. XRCC1-H expression correlated with longer mean survival in all patients (31.1 vs 19.33 months; $p<0.01$ ), and by treatment in the chemo-treated ( 35.4 vs 20.3 months; $\mathrm{p}=0.008)$ and non-treated groups of patients ( 28.8 vs 18.5 months; $\mathrm{p}=0.019)$. ERCC1-H correlated with longer mean survival ( 33.4 vs 24.9 months; $p=0.013$ ) in the group of chemo treated patients, but did not correlate with outcome in the non-treated group.

Conclusions: High expression of XRCC1 and ERCC1 are associated with longer survival in biliary cancer, and this may be improved with adjuvant chemotherapy. This warrants further study in larger series.

1856 Duodenal Invasion Is Under-Staged in Distal Bile Duct Carcinoma Staging of the $7^{\text {th }}$ AJCC Cancer Staging System

K-W Min, S An, HJ Kang, SC Kim, K-M Park, Y-J Lee, E Yu, S-M Hong. Asan Medical Center, University of Ulsan College of Medicine, Seoul, Republic of Korea.

Background: One of the main changes of the distal bile duct cancer staging scheme of the $7^{\text {th }}$ American Joint Committee on Cancer (AJCC) cancer staging system is downstaging of duodenal invasion from pT4 to pT3 classification along with pancreas and gallbladder invasions without enough validation studies. 
Design: Two hundred eighty-six cases with surgically resected distal bile duct carcinomas from 1991 to 2004 were selected and compared survival time of distal bile duct cancer patients according to organ invasion status, including pancreas and/ or duodenal invasion.

Results: One hundred eighty cases $(62.9 \%)$ of distal bile duct carcinomas were invaded into adjacent organs with 143 cases $(79.4 \%$ ) of single organ invasion (pancreas invasion, 140 cases; duodenum invasion, 2 cases, gall bladder invasion, 1 case) and 37 cases $(20.6 \%)$ of dual organ invasions (pancreas and duodenal invasion, 36 cases; gallbladder and duodenal invasion, 1 case). Cases with duodenal invasion (median, 17.1 months) had significantly worse patients' survival than those with pancreas invasion (median, 22.16 months) by both univariate $(\mathrm{p}=0.003)$ and multivariate $(\mathrm{p}=0.008)$ analyses. Conclusions: Duodenal invasion of distal bile duct carcinomas in the current $7^{\text {th }}$ AJCC cancer staging system based are under-staged on our observation. For better estimation of survival time in patients with surgically resected distal bile duct carcinomas, duodenal invasion should be up-staged in the new edition of the AJCC cancer staging system.

1857 Does Dissecting Acellular Stromal Mucin Associated with Intraductal Papillary Mucinous Neoplasms (IPMNs) Carry a Worse Prognosis?

JMorin, D Webber, O David, SE Kalloger, DF Schaeffer. University of British Columbia, Vancouver, BC, Canada.

Background: Intraductal papillary mucinous neoplasms (IPMNs), mucin-producing tumors arising within the pancreatic ductal system, are a recognized precursor lesion for pancreatic colloid carcinoma. Given the increased intraluminal pressure within the ductal system spillage of mucin into the extraductal stroma may occur. Although a diagnosis of colloid carcinoma arising within an IPMN is usually uncomplicated in the setting of neoplastic cells 'floating' within the mucin pools, the prognostic significance of acellular stromal mucin spillage has yet to be determined.

Design: Archival cases of IPMN ( $\mathrm{n}=27)$, resected at Vancouver General Hospital between 2006-2012, with associated clinical outcome data were reviewed and stratified into two groups: with (mIPMN; $n=10)$ and without (IPMN; $n=17$ ) extravasation of mucin into the stroma. Demographic information, histologic attributes considered to be associated with a higher potential of malignant transformation of IPMNs and follow-up data (range 1-6 yr) were compared between the two groups.

Results: IPMNs with stromal mucin extravasation (mIPMN) trended to occur at a later age (mean [yr] 68.1 vs 65.6), be larger in size (mean [cm] 3.04 vs 2.31) and were more commonly situated in the main pancreatic duct. No significant differences were identified in regards to histological grade and subtype (see figure 1). Overall survival (OS) at 1 and 2 year time points after initial diagnosis did not show any recurrences or death within either group.

\begin{tabular}{|l|c|c|c|}
\hline Variable & mIPMN (n=10) & $\begin{array}{c}\text { IPMN } \\
(\mathrm{n}=17)\end{array}$ & $p$-value \\
\hline Age (mean [yr]) & $68.1(47-81)$ & $65.6(51-86)$ & 0.54 \\
\hline Tumor size (mean [cm]) & 3.04 & 2.31 & 0.35 \\
\hline IPMN subtype & $7(70 \%)$ & $6(35 \%)$ & 0.13 \\
-Main duct & $1(10 \%)$ & $7(44 \%)$ & \\
--Branch duct & $2(20 \%)$ & $4(21 \%)$ & \\
-Mixed & $7(70 \%)$ & $10(63 \%)$ & 0.34 \\
\hline Histological subtype & $1(10 \%)$ & $5(31 \%)$ & \\
-Intestinal & $1(10 \%)$ & $2(6 \%)$ & \\
-Foveolar & $1(10 \%)$ & 0 & \\
-Pancreatobiliary & & & \\
-Mixed & $8(80 \%)$ & $15(88 \%)$ & \\
\hline Histological Grade & $2(20 \%)$ & $2(12 \%)$ & \\
-Low grade & & & \\
-High grade &
\end{tabular}

Conclusions: While mIPMNs possess histologic characteristics associated with a higher risk phenotype, such as main duct subtype and larger size, no difference in survival or recurrence rate was identified. Although this study is limited by the overall short term follow-up data and the cohort size, acellular mucin pools associated with IPMNs do not appear to carry a worse prognosis than those without.

1858 Low Nuclear Accumbens-1 Expression Is Related to Poor Prognosis in Pancreatic Ductal Adenocarcinoma; an Effect Opposite from Gynecological Carcinomas

T Nishi, R Maruyama, A Araki, N Ishikawa, T Urano, N Nakayama, K Nakayama, M Yoshida, Y Tajima. Shimane University School of Medicine, Izumo, Japan; Matsue Municipal Hospital, Matsue, Japan.

Background: Nucleus accumbens-associated protein 1 (NAC1) is overexpressed in various carcinomas including ovarian, cervical, breast, and pancreatic carcinomas. High expression of NAC1 is considered to have adverse effects on prognosis through negative regulation of growth arrest and DNA-damage-inducible 45 -interacting protein 1 (GADD45GIP1) in ovarian and cervical carcinomas. Recently we found that low expression of NAC1 was related to poor prognosis in pancreatic ductal adenocarcinoma (PDA), totally opposite effect from the result of gynecological cancers. We report the relationship between immunohistochemical expression of NAC1 in invasive PDA and clinicopathological parameters, and GADD45GIP1 expression and invasion assay after siRNA knockdown of NAC1 using pancreatic cancer cell lines.
Design: From 2000 to 2010, we retrieved 67 appropriate cases with typical invasive PDA. The median follow-up duration after surgical resection was 12.8 months (range: 0.3-72 months). The expression of NAC1 was measured using immunohistochemistry (IHC), and the IHC results were objectively analyzed by computer-assisted image analysis on the IHC-stained slides converted to whole slide images (as known as virtual slides) in order to investigate its correlation with various clinicopathological parameters and prognosis. The expression of GADD45GIP1 after knockdown of NAC1 of PDA cell lines was analyzed by Western blot, and invasion assay was also performed using Matrigel.

Results: Patients with low NAC1 PDA had worse overall survival $(\mathrm{P}=0.0010)$ and a shorter disease-free survival $(\mathrm{P}=0.0036)$ than patients with high NAC1 PDA. This was a clinical effect totally opposite from that reported in ovarian and cervical carcinomas. Low NAC1 group was correlated with more frequent venous invasion $(\mathrm{P}=0.047)$ and lymph node metastasis $(\mathrm{P}=0.0017)$ and higher UICC clinical stages $(\mathrm{P}=0.02)$ than high NAC1 group. Furthermore, knockdown of NAC1 in pancreatic carcinoma cell lines did not increase expression of the GADD45GIP1 protein. In addition to it, invasion assay showed significantly increased invasive ability of the cancer cells after knockdown of NAC1.

Conclusions: These results indicate that the gene(s) regulated by NAC1 vary depending on the types of carcinoma or originating tissue, and that low expression of NAC1 predicts poor prognosis for patients with PDA. This finding may help physicians choose appropriate treatment options and give patients proper information about their prognosis.

\section{Tumor Budding Is an Independent Adverse Prognostic Factor in} Pancreatic Ductal Adenocarcinoma

KM O'Connor, HH Li-Chang, SE Kalloger, DL Webber, DA Owen, DF Schaeffer. Vancouver General Hospital, Vancouver, BC, Canada.

Background: Although substantial progress has been made in our understanding of the biology of pancreatic cancer, pancreatic ductal adenocarcinoma (PDAC) remains the $4^{\text {th }}$ most common cause of cancer related death in North America. Tumor budding is a well-established adverse prognostic factor in colorectal cancer, and in this setting budding is associated with a lesser degree of peri-tumoral inflammation. However, the significance of tumor budding in PDAC is uncertain; one previous study has shown budding to be an independent prognostic factor. We aimed to assess the prognostic significance of tumor budding in PDAC, and to determine its relationship with peritumoral inflammation.

Design: Archival cases of PDAC $(n=192)$ were assessed for tumor budding using H\&E sections only; tumor buds were defined as single tumor cells or clusters consisting of $<5$ cells. The slide with the highest amount of budding was selected and assessed for the presence of high-grade budding ( $\geq 10$ buds/10 HPF) or low-grade budding $(<10$ buds/10HPF). The numbers of tumor infiltrating lymphocytes (TILs) were counted, and peri-tumoral lymphocytes and neutrophils were scored semi-quantitatively. These features were then correlated with baseline clinicopathologic features and outcome. Results: High-grade budding was present in 138/192 cases (72\%), low-grade budding was present in $32 / 192$ cases ( $17 \%$ ), and there was no budding in $22 / 192$ cases $(11 \%)$. The presence of budding was associated with inferior overall survival and there was no significant difference in overall survival between those with high- and low-grade budding. On multivariable analysis, tumor budding was prognostic independent of T-stage, grade, nodal status, lymphovascular and perineural invasion. Other independently prognostic variables included grade, and nodal status (Table 1). There was no significant relationship between tumor budding and the degree of peri-tumoral inflammation or number of TILs.

Table 1. Multivariable analysis for overall survival

Variable

Tumour Budding (present vs absent)

Grade (high [3] vs low [1\&2]

Extent (pT3 [

Lymph node metastasis (pN1 vs pNO)

Perineural invasion (present vs absent)

\begin{tabular}{|l|l|l|}
\hline $1.29(0.90-1.88)$ & 0.1713 \\
\hline
\end{tabular}

Conclusions: Tumor budding is independent adverse prognostic factor in PDAC. The assessment of budding can be performed on H\&E slides and may therefore be readily implemented into routine practice.

1860 "Carcinoma In-Situ" of the Gallbladder: The SEER Database Perspective

K Patel, S Balci, B Saka, J Knight, O Basturk, J Sarmiento, JC Roa, JC Araya, J Sweeney, P Terry, $M$ Goodman, V Adsay. Emory, Atlanta, GA; MSKCC, NY, NY; Frontera, Temuco, Chile; UT, Knoxville, TN.

Background: A previous analysis of carcinoma "in-situ" (CIS) of gallbladder (GB) reported to the NCI's Surveillance Epidemiology End Results (SEER) program demonstrated 5- and 10-yr survival estimates of $100 \%$ and $70 \%$, respectively. In our experience from high-incidence regions, early-gallbladder carcinoma, which also includes minimally invasive mucosa-confined carcinoma has a very good prognosis with 10 -yr survival of $90 \%$, if the GBs are sampled entirely and the possibility of a more advanced carcinoma has been carefully ruled out.

Design: 685 cases recorded as CIS of GB in the SEER database, 1973-2010, with survival information, were analyzed. In addition to observed survival, relative survival, which is more relevant to older population, was also calculated based on the expected survival in the general population with the same demographics. Cases with additional designation of "adenoma", "papillary" or "villous" were regarded as possibly representing tumoral forms of CIS (adenoma-associated or intracholecystic papillary neoplasm-associated) and grouped as "tumoral". 
Results: $80 \%$ of the patients were white, $69 \%$ were female, and almost half $(49 \%)$ were $\geq 70$ yrs old. Observed survival was $91 \%$ at $1-\mathrm{yr}, 80 \%$ at $3-\mathrm{yr}, 72 \%$ at $5-\mathrm{yr}, 53 \%$ at 10 -yr. The corresponding 1-, 3-, 5-, 10-yr estimates for relative survival were $94 \%$, $89 \%, 87 \%$ and $79 \%$, respectively. Survivals were generally slightly higher for "tumoral" cases compared to "flat" cases.

Survivals
\begin{tabular}{|l||l||l||l||l|}
\hline \hline & 1 yr (\%) & 3 yr (\%) & 5 yr (\%) & $10 \mathrm{yr}(\%)$ \\
\hline Observed & 91 & 80 & 72 & 53 \\
\hline Relative & 94 & 89 & 87 & 79 \\
\hline
\end{tabular}

Relative Survivals of Subgroups*

\begin{tabular}{|c|c|c|c|c|c|}
\hline & & $\sqrt{1 \text { yr (\%) }}$ & 3 yr (\%) & 5 yr (\%) & $\sqrt{10 \mathrm{yr}(\%)}$ \\
\hline \multirow[t]{2}{*}{ Gender } & Female & 95 & 91 & 89 & 80 \\
\hline & Male & 91 & 85 & 85 & 85 \\
\hline \multirow[t]{2}{*}{ Subtype } & Tumoral & 95 & 91 & 90 & 77 \\
\hline & Flat & 94 & 88 & 87 & 80 \\
\hline
\end{tabular}

Includes only cases with known gender \& type info

Conclusions: Cases recorded as "in-situ" carcinoma of GB in the SEER database are mostly elderly females. The 10 -yr survival of $79 \%$ is worse than what would be expected from the early-gallbladder carcinoma data from high-risk regions (10-yr survival, $90 \%$ ). This difference is likely attributable to under-sampling or underdiagnosis (GBs with CIS are submitted entirely in high-risk regions but not in the US). In particular, deaths within 1 year likely represent underdiagnosed higher-stage carcinomas. Of the deaths in longer $\mathrm{F} / \mathrm{U}$, some likely represent the true CIS cases, and as such may reflect a field-defect/effect phenomenon in the biliary tract. Tumoral CIS of GB may have slightly better prognosis. It is imperative for pathologists to examine GBs entirely to rule out advanced carcinomas, and for clinicians to place these patients in long-term $\mathrm{F} / \mathrm{U}$.

1861 Comparison of the Clinicopathologic Features of Short and Long Pancreatic Ductal Adenocarcinoma Survivors after Pancreaticoduodenectomy

$Z$ Ren, $N$ Hejazi, A Rashid, MH Katz, H Wang, RA Wolff, J Abbruzzese, JB Fleming, $H$ Wang. The University of Texas MD Anderson Cancer Center, Houston, TX.

Background: Pancreatic ductal adenocarcinoma (PDAC) remains one of the most lethal malignancies. Even for the patients who undergo pancreatectomy, the clinical courses and prognosis vary with survival ranging from few months to more than 10 years. The aim of this study is to compare the clinicopathologic factors associated with short and long survival after pancreatectomy.

Design: Our study population consists of 95 long survivors (survival $>5$ years after pancreatectomy) and 74 short survivor (survival $<1$ year after pancreatectomy) who underwent pancreatectomy at our institution. The H\&E slides from all cases were reviewed. The clinicopathologic data were obtained by reviewing the medical records, including age, gender, family and personal history of cancer, tumor stage, lymph node status, resection margin, lymphovascular and perineural invasion, and neoadjuvant therapy. Fisher's exact tests were used to determine the statistical significance.

Results: The long and short survivor groups were not statistically different for age (62.6 \pm 9.5 years vs $64.6 \pm 9.5$ years), gender ( $45.3 \%$ vs $37.8 \%$ females), neoadjuvant therapy $(67 \%$ vs $54 \%$ respectively, $\mathrm{P}=0.08$ ) and types of resection (pancreaticoduodenectomy $99 \%$ vs $93 \%$ respectively, $\mathrm{P}=0.09$ ). Long survivors had lower tumor ( $\mathrm{pT}$ ) and American Joint Committee on Cancer (AJCC) stage; lower frequencies of lymph node metastasis, positive resection margin, lymphovascular and perineural invasion; and lower recurrence rate than short survivors $(\mathrm{P}<0.05)$. During the follow-up, 10 patients in long survivor group developed a second primary tumor including two patients with second PDAC in the remaining pancreas.

Conclusions: Our study showed that histopathological features such as pT and AJCC stages, lymph node metastasis, positive resection margin, lymphovascular and perineural invasion are associated with patient survival after pancreatectomy.

$1862 \quad$ Not All Combined Intraductal Papillary Mucinous Neoplasms Behave like Main-Duct Lesions: Implications of Minimal Involvement of the Main Pancreatic Duct

K Sahora, C Fernandez-del Castillo, F Dong, G Marchegiani, M Mino-Kenudson. Massachusetts General Hospital, Boston, MA.

Background: The malignant potential of IPMN is closely associated with involvement of the main pancreatic duct (MPD). Because neoplasms involving both main and side branches (combined IPMN) are thought to have the same malignant potential as that of main duct (MD)-IPMN, resection is recommended by the international guidelines. However, the biological nature of combined IPMN with only minimal involvement of MPD (min-com-IPMN) may be different.

Design: A prospective database of 407 resected IPMNs was queried to identify combined and branch duct (BD)-IPMNs. Minimal involvement of MPD was defined as absence of gross abnormalities (except for dilatation) of MPD and non-circumferential microscopic involvement of MPD limited to few sections. Clinicopathological features and patient outcomes were compared between min-com-IPMN and other groups.

Results: Of 407 lesions, we identified 45 min-com-IPMN, 126 IPMN with extensive involvement of MPD (ex-com-IPMN) and173 BD-IPMN. The majority of min-comIPMNs were incidentally found and increased cyst size on surveillance was the leading indication for resection. The median diameter of the MPD was $3 \mathrm{~mm}$ in min-com-IPMN vs. $9 \mathrm{~mm}$ in ex-com-IPMN $(\mathrm{p}=<.0001)$ and $2 \mathrm{~mm}$ in BD-IPMN $(\mathrm{p}=<.0001)$. Cysts $\geq$ $10 \mathrm{~mm}$ were present in $64 \%$ of ex-com-IPMNs versus $96 \%$ of min-com-IPMNs $(\mathrm{p}=$ $<.0001)$. Most significantly, the vast majority of min-com-IPMN $(90 \%)$ exhibited gastric-type epithelium, similar to BD-IPMN, while intestinal-type epithelium was present in $48 \%$ of ex-com-IPMNs $(\mathrm{p}=<.0001)$. Upon evaluating the epithelial histology of MPD vs. branch ducts in min-com-IPMN, MPD was exclusively involved by gastric-type epithelium. The main-duct components harbored lower or the same grade of dysplasia as the cystic lesion / branch-duct components and no high-grade dysplastic epithelium was seen in MPD. The overall prevalence of high-grade dysplasia ( $11 \% \mathrm{vs.}$ $41 \%)$ and invasive carcinoma $(6.7 \%$ vs. $32 \%)$ was significantly lower in min-comIPMN compared to ex-com-IPMN $(\mathrm{p}<.0001)$. These differences were reflected in better disease specific outcomes of min-com-IPMNs compared to ex-com-IPMNs ( $\mathrm{p}=0.051)$. Conclusions: min-com-IPMN often presents with no MPD dilation and is an incidental finding by microscopic examination. It shares the pathological features and less aggressive biology of BD-IPMN. We propose that min-com-IPMN be categorized and treated differently than ex-com-IPMN.

\section{Proposal for a New and Prognostically Valuable Tumor Size} Based T-Stage for Pancreatic Adenocarcinoma

B Saka, S Balci, P Bagci, S Maithel, D Kooby, J Sarmiento, C Staley, B El-Rayes, $H$ Choi, J Knight, $M$ Goodman, A Krasinskas, V Adsay. Emory, Atlanta, GA.

Background: The current AJCC T-staging system for pancreatic ductal adenocarcinoma lacks prognostic value in most studies unless selective grouping of different T-stages performed. Our aim was to develop a more clinically relevant T-classification system. Design: The clinical relevance of the AJCC parameters was analyzed in 250 consecutive pancreaticoduodenectomy specimens for PDAC

Results: There was no prognostic correlation with the current AJCC/UICC staging protocol $(\mathrm{p}=0.48$; table 1$)$. No survival correlation was identified with the individual parameters by univariate analysis either: Carcinoma extension into "peripancreatic soft tissue", which was found in $89 \%$ of the cases did not have any correlation with clinical outcome $(\mathrm{p}=0.83)$, nor did involvement of duodenum $(\mathrm{p}=0.76)$ or ampulla $(\mathrm{p}=0.45)$ or extra-pancreatic CBD $(\mathrm{p}=0.91)$. When a size-based T-stage protocol partially similar to the one used by ENETS for PanNETs was employed (with $\leq 2 \mathrm{~cm}$ as T1, 2-4 as T2, and $>4$ as T3), a statistically significant correlation with survival was found $(\mathrm{p}=0.0011$, Figure 1$)$.

Current T Stage (According AJCC 7th) and Survivals

\begin{tabular}{|l||l||l||l|l||}
\hline T Stage According to AJCC 7th & $n(\%)$ & 3 year survival $(\%)$ & 5 year survival $(\%)$ & p- value \\
\hline pT1 & $6(2.4)$ & 20.0 & 20.0 & 0.48 \\
\hline pT2 & $4(1.6)$ & 0.0 & 0.0 & \\
\hline PT3 & $234(95.1)$ & 27.5 & 16.3 & \\
\hline pT4 & $2(0.8)$ & 0.0 & 0.0 & \\
\hline
\end{tabular}

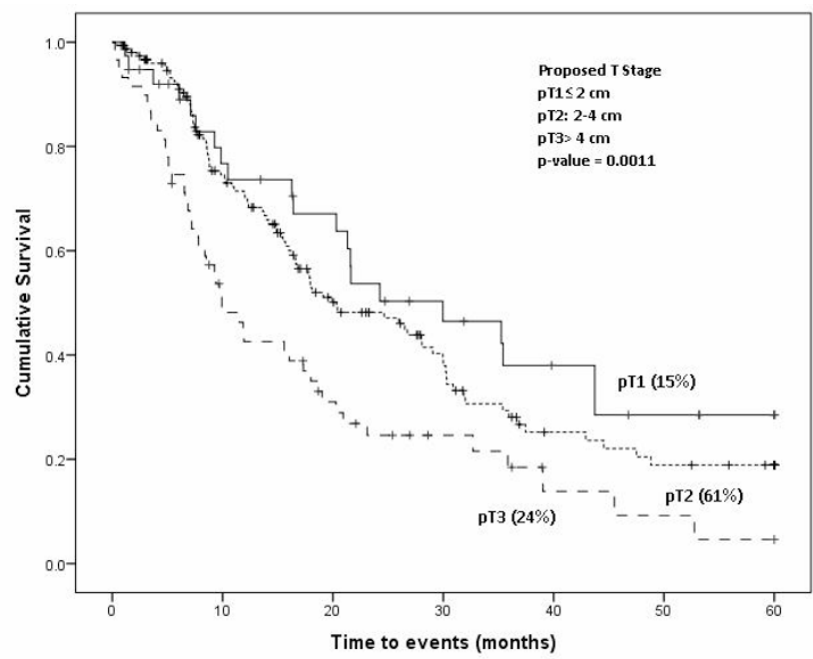

Conclusions: This study confirms in a large cohort of carefully reviewed cases that the current AJCC/UICC T-stage does not have prognostic relevance, and that the individual parameters other than size used in this system are also irrelevant. The sizebased T-stage system proposed here, potentially has greater prognostic value and is more clinically relevant.

1864 Tumoral (Flat) Gallbladder Dysplasia

B Saka, S Balci, N Dursun, P Bagci, K-T Jang, JC Roa, P Terry, I Genco, O Basturk, $V$ Adsay. Emory, Atlanta, GA; SMC, Seoul, Korea; PUC, Santiago, Chile; UT, Knoxville, TN; MSKCC, NY, NY.

Background: Various cell lineages are now being recognized in gallbladder (GB) neoplasia. There is virtually no data on the immunoprofile of non-tumoral (flat) dysplasia/intraepithelial-neoplasia subtypes.

Design: IHC profile of biliary-cuboidal, biliary-pencillate, gastric, \& intestinal lineages of non-tumoral dysplasia was investigated and was contrasted with that of normal and other neoplastic GB.

Results: Biliary markers: MUC1, absent in normal mucosa and present in $70 \%$ of invasive ca, was detected in $20 \%$ of dysplasias, mostly in the biliary-cuboidal. Gastric markers: MUC5AC \& MUC6, which were commonly (62 \& 64\%) detected in normal GB mucosa, was less common ( 35 \& $11 \%$ ) in dysplasia, with the exception of substantial (75\%) MUC5AC in gastric. Intestinal markers: While CDX2 expression was absent in normal GB mucosa (except for rare metaplastic goblet cells), its aberrant expression was detected in dysplasia; lowest (13\%) in biliary-cuboidal, highest $(80 \%)$ in intestinal. MUC2 also highlighted the goblet cells in most dysplasia, but not seen in non-goblet 
cells of dysplasia, including intestinal type. CD10, which typically formed a fairly continuous apical band in $88 \%$ of normal GB, and was very low (17\%) in invasive ca, was often interrupted or lost in the dysplasias, lowest $(8 \%)$ in biliary-cuboidal and highest (75\%) in biliary-pencillate. CK20, absent in normal mucosa, could be expressed aberrantly in dysplasia; metaplastic (gastric and intestinal) subtypes showed CK20 in all examples, biliary ones were all negative. Biliary-pencillate type, which had overlapping histology with intestinal, more commonly expressed biliary markers (CK7:100 vs 50\%; CA19-9: 75 vs $50 \%$; CEA: 82 vs 50\%), and mostly lacked intestinal ones (CK20: 0 vs $100 \%$; CDX2: 37 vs $80 \%$ ).

Table 1:

\begin{tabular}{|c|c|c|c|c|c|c|c|c|c|c|}
\hline Normal(n=26) & $\sqrt{101}$ & 10 & 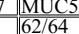 & MUC6 & 0 & & & & $\frac{\frac{C L}{28}}{28}$ & \\
\hline $\begin{array}{l}\text { Non-tumoral } \\
\operatorname{dysp}(n=110)\end{array}$ & 20 & 71 & $35 / 11$ & & $3 / 17 / 12$ & & 43 & & 80 & 87 \\
\hline $\begin{array}{l}\text { Tumoral } \\
\text { dsyp(ICPN, } \\
n=74)\end{array}$ & 18 & 62 & $39 / 26$ & & $12 / 14 / 13$ & & & & & \\
\hline Inv. ca(n=95) & $\sqrt{69}$ & 884 & $\sqrt{35 / 8}$ & & $10 / 23 / 18$ & & 17 & & 65 & 65 \\
\hline
\end{tabular}

\section{Inv. ca}

\begin{tabular}{|c|c|c|c|c|c|c|c|}
\hline & $\underline{\mathrm{MU}}$ & CK & MUC5AC/MUC6 & MUC2/CL & CD & CE & CA19-9 \\
\hline 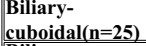 & 22 & 83 & $35 / 12$ & $0 / 13 / 0$ & 8 & 62 & 76 \\
\hline $\begin{array}{l}\text { Biliary- } \\
\text { pencillate( }(n=15)\end{array}$ & 0 & 100 & $30 / 13$ & $\mid 0 / 37 / 0$ & 75 & 75 & 81 \\
\hline Gastric(n=25) & 0 & 100 & $75 / 19$ & $77 / 31 / 100$ & 20 & 76 & 82 \\
\hline Intestinal(n=8) & 14 & ] & [57/25 & {$[0 / 80 / 100$} & ]60 & ]50 & 50 \\
\hline
\end{tabular}

Conclusions: Dysplasia in the GB is associated with immunophenotypic alterations different from normal, some confirming its similarities \& precursor nature to invasive carcinoma. IHC findings also support the presence of different cell lineages with different profiles, albeit with overlaps, in dysplasia.

1865 Will the pT1 and pT2 Pancreas Cancer Please Stand Up? "Peripancreatic Soft Tissue" Is Involved in Most Pancreatic Ductal Adenocarcinomas (PDAC), Negating Its Value as a Staging Parameter and Necessitating a New Staging Scheme

B Saka, I Oliva, S Bandyopadhyay, O Basturk, S Balci, S Maithel, D Kooby, J Sarmiento, C Staley, B El-Rayes, H Choi, J Knight, A Krasinskas, V Adsay. Emory, Atlanta, GA; WSU, Detroit, MI; MSKCC, NY, NY.

Background: The pancreas does not have a capsule, its lobules are irregularly distributed, intraparenchymal adipose tissue is abundant, and the soft tissue-pancreas interface is often obscured by pancreatitis or peritumoral changes. Thus, documentation of peripancreatic soft tissue (PST) involvement (pT3 per AJCC) has been challenging and highly variable, possibly contributing to the lack of prognostic relevance for T-stage in most major studies.

Design: In 250 consecutive pancreaticoduodenectomies with PDAC, fragments of soft tissue covering the pancreatic head (true PST) were examined. Briefly, in this orangepeel approach, the soft tissue around the pancreatic head is shaved-off in 7 arbitrary regions before any sections are taken and at least 1 casette is submitted from each region even if no lymph nodes are identified.

Results: Carcinoma foci were identified in these orange-peeled soft tissues, in $223 / 250(89 \%)$ of the cases. An additional $4.4 \%$ qualified as pT3 based on extension into "adjacent" sites (duodenum/ampulla/extra-pancreatic CBD). Only 4\% qualified as pT1 and pT2. AJCC T-stage defined as such was not found to have any correlation with survival (Figure 1). PST involvement often appeared as isolated solitary ducts mimicking PanINs, was not appreciated grossly, and was often identified in unsuspected regions; even the anterior region, which is not routinely sampled in conventional grossing protocols, was involved in $32 \%$ of cases. The frequency of involvement of different regions was: Anterior $27.6 \%$, anterior pancreaticoduodenal $15.3 \%$, superior $19 \%$, inferior $16 \%$, posterior pancreatic $23.4 \%$, posterior pancreaticoduodenal $19 \%$, uncinate $57.6 \%$. Two-thirds of the cases had $>1$ surfaces involved.

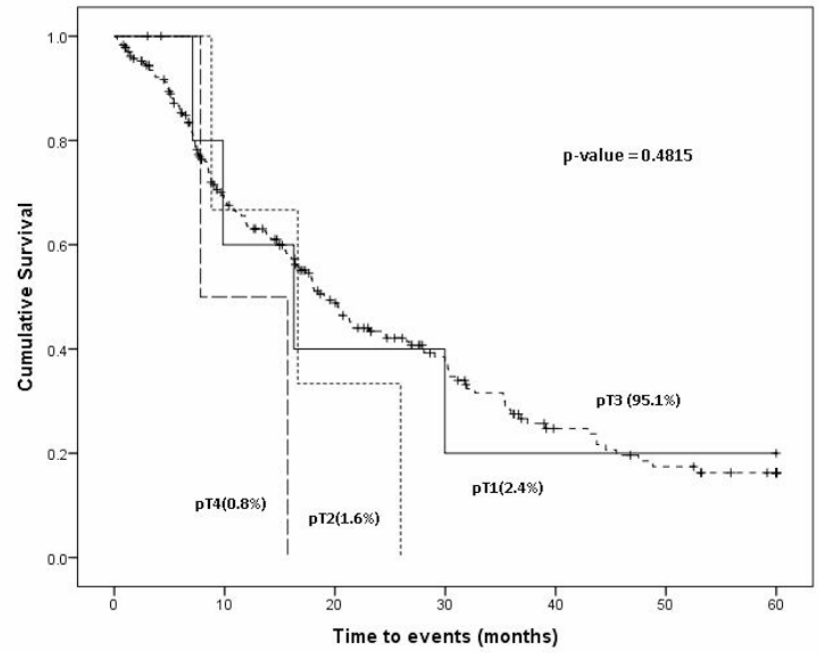

Conclusions: This study confirms that PDACs are typically spread far beyond what meets the eye at the time of diagnosis, extending to various aspects of PST and the current definition of "PST involvement=pT3" allows only $4 \%$ of PDACs to truly qualify as pT1 or T2. More importantly, the current staging protocol does not correlate with prognosis. A more clinically relevant staging protocol is needed for PDAC.

1866 Diagnostic Criteria for Intraductal (Intracystic) Papillary Neoplasm of the Bile Duct (IPNB) and the Gallbladder (ICPN): Histological and Immunohistochemical (IHC) Evaluation of $\mathbf{5 0}$ Cases

D Sato, T Mitsuhashi, Y Hatanaka, Y Nakanishi, S Hirano, Y Matsuno. Hokkaido University Hospital, Sapporo, Japan.

Background: IPNB and ICPN are characterized by intraductal (intracystic) papillary growth and have shown better outcome compared with the more common nodularsclerosing carcinoma. IPNB is a recognized precursor of invasive carcinoma, but its pathogenesis and the diagnostic criteria rather than intraductal papillary growth is ill-defined. They may represent a carcinogenesis pathway different from that of conventional bile duct carcinomas arising from flat dysplasia. This study aimed to examine the clinicopathologic features and outcomes of IPNB and ICPN, and to evaluate the diagnostic criteria.

Design: Fifty cases with histologic papillary features were included in this study [10 hilar, 10 intrahepatic, and 22 distal bile ducts and 11 gallbladder, $\mathrm{M}: \mathrm{F}=30: 20$, median age 70 (46-86)]. Histopathologic and IHC findings for mucin core (MUC) 1, 2, 5AC and 6 proteins and p53 were used to subclassify IPNBs and ICPNs. In addition, cluster analysis using MUC proteins was performed. Prognostic data with 5-year survival rate were analyzed and correlated with clinical and pathologic parameters.

Results: Histopathologic examination revealed 31 pancreatobiliary, 7 gastric, 7 intestinal, and 5 oncocytic subtypes. Fifteen 553 -positive cases showed poorer prognosis than those of negative cases $[43 \%$ and $64 \%$, respectively $(\mathrm{p}=0.016)]$. MUC1+ cases showed poor prognosis $(\mathrm{P}=0.014)$. Cluster analysis using MUC proteins divided those tumors into 9 subgroups.

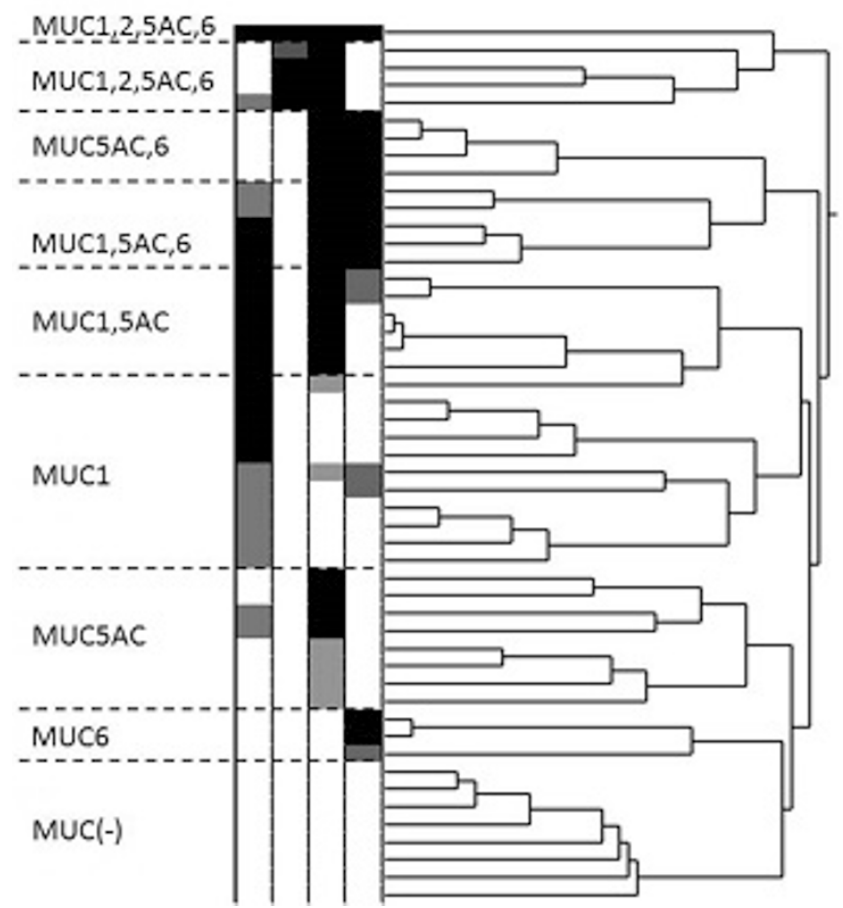

MUC2+MUC5+ tumors were all intestinal-type histologically; however, other groups failed to show histological correlation. According to the cluster analysis, the MUC2+MUC5+ group (5 cases) was separated from other 45 cases, and showed a tendency of better prognosis [ $75 \%$ and $45 \%$, respectively $(\mathrm{p}=0.24)]$.

Conclusions: Although IPNB and ICPN share some histologic and clinical features with intraductal papillary neoplasm of the pancreas, IHC analysis of MUC proteins might have a limited value to subclassify IPNBs and IPCNs. Further studies are in progress including stem cell markers to establish the diagnostic criteria of IPNBs and IPCNs from the perspective tumorigenesis.

1867 Molecular Diagnostic, Prognostic and Therapeutic Role of miR-221 in Pancreatic Cancer

S Sethi, S Sarkar, H Dubaybo, S Ali, P Goncalves, S Kollepara, PA Philip, Y Li. Wayne State University, Detroit, MI.

Background: Comprehensive molecular profiling of microRNAs (miRNAs) and subsequent target gene analysis of pancreatic cancer $(\mathrm{PaCa})$ can provide tumor specific miRNAs signatures to improve diagnostic accuracy, refine prognostic and predictive capabilities, and may also serve as therapeutic targets. In PaCa such a comprehensive analysis has not been reported.

Design: RNA extracted from scant amounts of formalin fixed paraffin embedded $\mathrm{PaCa}$ tumor and normal pancreatic tissues were profiled for miRNA expression using microfluidic biochip microarrays (which interrogate 2019 unique mature miRNAs). 
The expression of abnormal miRNAs was then validated and quantified using real time RT-PCR. Data was statistically analyzed using the Student's t-test. Survival data obtained in each case was correlated with the miRNA data using Kaplan-Meier analysis. Oncogenic potential, therapeutic modulation and downstream target genes of altered miRNA were evaluated by real time RT-PCR using PaCa cells for mRNA expression. Results: miRNA profiling showed high expression of miR-221 in PaCa tissues compared to normal pancreas $(\mathrm{p}=0.001)$. These findings were validated and quantified using qRT- PCR ( $\mathrm{p}=0.0029)$ (Figure 1A)

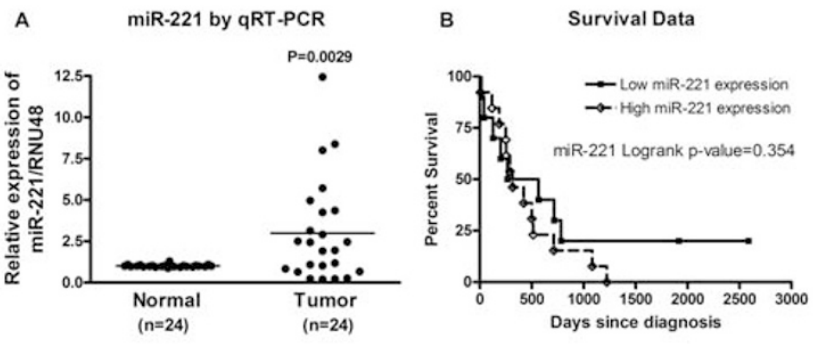

Survival analysis using Kaplan-Meier, demonstrated shorter survival of patients with higher expression of miR-221 compared to those with relatively lower levels of miR221 (Figure1B). The cell proliferation index of PaCa cells was increased by miR-221 mimics transfection and decreased by miR-221 inhibitors. The predicted target genes of miR-221 identified by RT-PCR were PTEN, $p 27^{k i p l}, p 57^{k i p 2}$ and PUMA.

Conclusions: High throughput miRNA expression profiling showed high expression of miR-221 in PaCa tissues. Higher levels of miR-221 demonstrated poorer prognosis, suggesting the oncogenic potential of miR-221 in PaCa. Its target genes are PTEN, $p 27^{k i p 1}, p 57^{k i p 2}$ and $P U M A$ which are tumor suppressors, and found to be deregulated by over-expression or under-expression of miR-221 transfection studies in PaCa cells. These results provide molecular evidence of oncogenic potential of miR-221 in $\mathrm{PaCa}$ which may have a significant clinical impact on prognosis \& risk stratification and in designing novel targeted molecular therapeutics in the future to achieve the goal of personalized and precision medicine.

1868 Immunolabeling for LEF-1 Aids in the Diagnosis of SolidPseudopapillary Neoplasms of the Pancreas

AD Singhi, M Lilo, RH Hruban, KL Cressman, K Fuhrer, RR Seethala. University of Pittsburgh Medical Center, Pittsburgh, PA; Johns Hopkins Hospital, Baltimore, MD. Background: Solid-pseudopapillary neoplasms (SPNs) are rare pancreatic tumors of low-malignant potential and enigmatic origin. While they may mimic other pancreatic neoplasms, SPNs are often associated with gain-of-function mutations in the $\beta$-catenin gene. Such mutations are typically not observed in other pancreatic tumors and result in accumulation of $\beta$-catenin. $\beta$-catenin is a central component of the Wnt signaling pathway and mediates gene expression through the lymphoid enhancer factor and T-cell factor transcription (LEF-1/TCF) complex. As LEF-1 has not been explored in SPNs or other pancreatic tumors, we analyzed both LEF-1 and $\beta$-catenin protein expression by immunohistochemistry in cytologic and surgical resection specimens.

Design: Immunohistochemical labeling for LEF-1 and $\beta$-catenin was performed on 111 surgical resected pancreatic neoplasms: 27 SPNs, 44 well-differentiated pancreatic neuroendocrine tumors (PanNETs), 2 high-grade pancreatic neuroendocrine carcinomas (PanNECs), 25 pancreatic ductal adenocarcinomas (PDACs), 9 acinar cell carcinomas (ACCs) and 4 pancreatoblastomas (PBs). Corresponding preoperative cytopathology cell blocks from 8 SPNs, 29 well-differentiated PanNETs, 1 high-grade PanNEC, 12 PDACs and 2 ACCs were also immunolabeled.

Results: All 27 resected SPNs and all 8 SPN cytology samples had strong and diffuse nuclear labeling for LEF-1.The surrounding uninvolved pancreatic parenchyma in the surgical samples, including the exocrine and endocrine components, were devoid of any labeling. Similarly, both nuclear and cytoplasmic accumulation of $\beta$-catenin was identified in all 27 surgical SPNs and all 8 SPN cytology samples. Labeling for LEF-1 and $\beta$-catenin was absent in all well-differentiated PanNETs, high-grade PanNECs, PDACs, and ACCs studied. However, nuclear LEF-1 and $\beta$-catenin labeling was detected in all 4 PBs with expression ranging from $10-40 \%$ of the neoplastic cells. LEF-1 and $\beta$-catenin labeling was primarily centered around and within squamoid corpuscles including tumor cells with optically clear nuclei which are known to contain biotin.

Conclusions: Abnormal nuclear $\beta$-catenin accumulation is accompanied by nuclear LEF-1 overexpression in both SPNs and PBs. However, in contrast to PBs, a diffuse, nuclear labeling was observed in SPNs. In conjunction with $\beta$-catenin, LEF-1 is a useful ancillary stain in the diagnosis of SPNs.

\section{Frozen Section Evaluation of Margins for Pancreatectomy}

F Smith-Chakmakova, P Navarro-Alvarez, Z Yang. Penn State Hershey Medical Center, Hershey, PA

Background: Intraoperative assessment of pancreatic and bile duct margins is a routine practice for pancreatectomies in most hospital centers. However, these are difficult frozen sections (FS) and their misinterpretation is one of the major complaints we receive from the surgeons. As part of the quality control process, we sought to retrospectively review our performance within the last three years, in order to identify opportunities for improvement and also assess the impact on patient survival.

Design: A retrospective database review was performed for all patients who underwent proximal, distal, and total pancreatectomy procedures for carcinomas from 2010 to 2013. The pathology reports and clinical information were reviewed. The original FS and permanent sections for bile duct and pancreatic margins were examined side by side.
Patient survival information was obtained from social security death index database. Results: Seventy-eight pancreatectomies cases ( 66 proximal, 9 distal, and 3 total) with a final diagnosis of invasive carcinoma were retrieved, which included 52 cases from pancreas, 12 from ampulla, 4 from duodenum, and 5 from distal bile duct. In total there were 85 FS for pancreatic margin and 67 FS for bile duct margin. Discordance between FS versus final diagnosis was seen in 15 patients (14 pancreatic carcinoma, 1 duodenal carcinoma) and all of them were positive at final diagnosis. These includes 10 pancreatic margin FS (11.8\%) and 5 common bile duct margin FS (7.5\%), which showed no difference in the distribution of misinterpretation (Fisher's exact test, $p>0.5$ ). Of the above 15 patients, 7 had a positive uncinate margin which was not sent for frozen, and 11 had lymph node metastases. Reasons for discordance include: nondiagnostic FS due to artifact ( 5 cases, $33 \%$ ), misinterpretation of the original FS ( 3 cases, $20 \%$ ), tumor present on deeper levels but not on original FS ( 2 cases, $13 \%$ ), and indeterminate ( 5 cases, 33\%). Using Kaplan-Meier survival analysis, there was no statistical difference in disease-specific survival between positive and negative final margin status in patients with either node-negative or node-positive pancreatic carcinoma (Logrank $p>0.25$ ). Conclusions: Our discrepancy rate in the interpretation of margins of pancreatectomy specimen during operation is well within the reported range. The most common reason of discordance was nondiagnostic FS due to artifact, followed by misinterpretation and undersampling. Although a misinterpreted margin may generate dissatisfaction from the surgeon and patients and may incur additional treatment, it does not seem to affect survival in our patient population.

\section{Invasive IPMN: Two Distinct Patterns of Microscopic Invasion with} Different Biology

JF Solus, L Fazlollahi, C Fernandez-del Castillo, G Marchegiani, MB Pitman, M Mino-Kenudson. Massachusetts General Hospital, Boston, MA.

Background: Microscopic foci of invasive carcinoma are not infrequently seen in intraductal papillary mucinous neoplasm (IPMN); however, little is known about patterns of microscopic invasion and their association with histopathological features and biology of IPMN.

Design: A prospective database of IPMNs was queried to identify invasive IPMNs resected after 2002 when extensive sampling of specimens was implemented. Each case was evaluated for histopathologic features of the intraductal and invasive components. The former included epithelial types, and the latter included macroscopic vs. microscopic invasion, number and maximum size of invasive foci, relation to the IPMN lesion, node involvement (LNI), lymphovascular (LVI), and perineural invasion (PNI). Microscopic invasion was defined as $<1 \mathrm{~cm}$ of the maximal invasive component.

Results: We identified 40 invasive IPMNs including 22 microscopic ones. Three patterns of invasion were seen: mass-forming invasion (type $1, n=17$ ), microscopic invasion closely associated with the main IPMN lesion (type 2, n=18), and microscopic invasion from small ducts away from the main IPMN lesion (type $3, n=5$ ). Type 2 invasion showed foci originating from lesional (main and/or branch) ducts within the parenchyma grossly affected by IPMN, while type 3 invasion showed microscopic foci from small ducts with high-grade dysplastic epithelium, located away from the main IPMN lesion and in the background of near normal parenchyma (likely via "cancerization" of pancreatic ducts). Histopathologic features of the 3 types are shown in Table 1.

Histological characteristics of invasion in IPMN
\begin{tabular}{|l|l|l||l||}
\hline \hline & Type 1 $(\mathrm{n}=17)$ & Type 2 $(\mathrm{n}=18)$ & yype $3(\mathrm{n}=5)$ \\
\hline Gastric/Intestinal/PB/Oncocytic & $4 / 9 / 5 / 0$ & $5 / 9 / 0 / 4$ & $1 / 0 / 4 / 0$ \\
\hline Tubular/Colloid/Oncocytic & $11 / 8 / 0$ & $11 / 5 / 4$ & $5 / 0 / 0$ \\
\hline Size (cm) & 4.1 & 0.2 & 0.28 \\
\hline \hline of invasive foci & 1 & 3.4 & 7.4 \\
\hline LNI (\%) & 41 & 5.6 & 40 \\
\hline LVI (\%) & 59 & 5.6 & 80 \\
\hline PNI (\%) & 59 & 5.6 & 60 \\
\hline
\end{tabular}

Type 2 invasion was rarely associated with LNI, LVI, PNI, and all oncocytic-type invasive IPMN belonged to this group. Type 3 invasion, often with multiple microscopic foci, consisted only of tubular adenocarcinoma arising from pancreatobiliary or highgrade gastric-type IPMNs and showed as aggressive features as type 1 invasion.

Conclusions: There are two patterns of microscopic invasion in IPMN, each associated with unique histopathologic features. Notably, type 3 invasion, associated with aggressive biology, consisted of multiple small foci in the background of near normal parenchyma. The results emphasize the importance of extensive sampling and thorough microscopic examination in IPMN.

1871 Pancreatic Neuroendocrine Tumors Express the RKIP Associated Pathways NFkappaB and MAP Kinase

BJ Swanson, LA Shirley, M Bloomston, WL Frankel. The Ohio State Wexner Medical Center, Columbus, $\mathrm{OH}$.

Background: Pancreatic neuroendocrine tumors (PNET) are a group of neoplasms whose metastatic potential and lethality can be difficult to predict. We have previously reported that Raf kinase inhibitory protein (RKIP) may be a metastasis suppressor in PNET. Here, we study down-stream signaling of RKIP in both the NFkappaB (NFKB) and MAP kinase (MAPK) signaling pathways of PNET.

Design: Tissue microarrays (TMAs) of PNET $(n=69)$ were constructed from archived samples. These TMAs consisted of both primary $(\mathrm{n}=57)$ as well as paired and unpaired metastatic samples $(n=12)$. TMAs were immunohistochemically stained for total NFKB (TNFKB), active NFKB (ANFKB), total MAPK (TMAPK) or active MAPK (AMAPK) and scored as strongly positive ( $3+$ ), moderately positive $(2+)$, weakly positive $(1+)$ or negative. 
Results: There were no statistically significant differences in expression of TNFKB, ANFKB, TMAPK, and AMAPK between primary and metastatic PNET as seen in table 1. As illustrated in table 2, TMAPK was positively correlated with RKIP while TNFKB was inversely correlated with mitotic count.

\begin{tabular}{|c|c|c|c|c|c|}
\hline \multirow[b]{2}{*}{ Protein } & \multirow{2}{*}{ Site } & \multicolumn{4}{|l|}{ Score } \\
\hline & & $3+$ & $2+$ & $1+$ & $\sqrt{0}$ \\
\hline TNFKB & Primary & $28 \%(15 / 53)$ & $43 \%(23 / 53)$ & $13 \%(7 / 53)$ & $15 \%(8 / 53)$ \\
\hline TNFKB & Metastasis & $17 \%(2 / 12)$ & $25 \%(3 / 12)$ & $33 \%(4 / 12)$ & $25 \%(3 / 12)$ \\
\hline ANFKB & Primary & $14 \%(7 / 51)$ & $22 \%(11 / 51)$ & $8 \%(4 / 51)$ & $57 \%(29 / 51)$ \\
\hline ANFKB & Metastasis & 0 & $10 \%(1 / 10)$ & $30 \%(3 / 10)$ & $60 \%(6 / 10)$ \\
\hline TMAPK & Primary & $93 \%(50 / 54)$ & $6 \%(3 / 54)$ & $2 \%(1 / 54)$ & 0 \\
\hline TMAPK & Metastasis & $91 \%(10 / 11)$ & $9 \%(1 / 11)$ & 0 & 0 \\
\hline AMAPK & Primary & $26 \%(14 / 54)$ & 0 & 0 & $74 \%(40 / 54)$ \\
\hline AMAPK & Metastasis & 0 & 0 & 0 & $100 \%(11 / 11)$ \\
\hline
\end{tabular}

Table 2. Correlation of TNFKB, ANFKB, TMAPK and AMAPK with Clinical and Pathologic

\begin{tabular}{|c|c|c|c|c|}
\hline Parameters & & & & \\
\hline & TNFKB & ANFKB & TMAPK & AMAPK \\
\hline RKIP & $\mathrm{p}$ & $\mathrm{p}$ & $\mathrm{p}$ & $\mathrm{p}$ \\
\hline & 0.405 & 0.386 & $0.001 *$ & 0.932 \\
\hline Mitotic count & $0.034^{*}$ & 0.509 & 0.124 & 0.953 \\
\hline Ki-67 & 0.481 & 0.451 & 0.217 & 0.978 \\
\hline Tumor size & 0.145 & 0.671 & 0.990 & 0.557 \\
\hline Presence of metastasis & 0.380 & 0.671 & 0.819 & 0.066 \\
\hline Length of survival & 0.224 & 0.453 & 0.556 & 0.759 \\
\hline
\end{tabular}
Length of survival

*-Statistically Significant

Conclusions: The NFkappaB and MAP kinase pathways are expressed in a subset of PNET. Furthermore, RKIP may work through the MAP kinase pathway. These results suggest a role for targeted therapy of the MAP kinase pathway in PNET.

1872 The Utility of Phosphohistone H3 Immunostain in the Assessment of Mitotic Rate and Histologic Grade of Pancreatic Well-Differentiated Neuroendocrine Tumors

SM Voss, MP Riley, P Lokhandwala, NJ Gusani, Z Yang. Penn State Milton S. Hershey Medical Center, Hershey, PA.

Background: Grading of pancreatic well-differentiated neuroendocrine tumors (WDNET) is based on mitotic rate or Ki67 labeling index. The cutoff on mitosis is narrow ( 2 mitoses $/ 2 \mathrm{~mm}^{2}$ ) but has significant prognostic value. Accurate assessment of the mitotic rate generally requires 50 high power fields (HPFs) which takes significant time and carries poor interobserver reproducibility. Phosphohistone H3 (PHH3) has been shown to be a specific marker for mitosis. This study aims to assess utility of PHH3 antibody in pancreatic WDNET.

Design: Forty cases $(\mathrm{M} / \mathrm{F}=0.8$, age $20-80$, median 50 ) of pancreatic WDNET were retrieved from the anatomic pathology archive. Immunostain for $\mathrm{PHH} 3$ was performed on representative tumor sections. Mitotic rate was assessed by four readers on HE and PHH3 stains. In addition, PHH3 stain was assessed in highly labeled fields (hotspot) on single low power (10x) and intermediate power (20x) by the senior author. Statistical analysis was performed using MedCalc version 12.7.4

Results: Based on mitotic rate on HE, there were $28 \mathrm{G} 1$ and $12 \mathrm{G} 2$ tumors, and mitotic rate ranged from 0 to 6.5 mitoses $/ 2 \mathrm{~mm}^{2}$. Intraclass correlation was 0.49 (single measure) based on the raw mitotic count. The Fleiss' kappa correlation for grade assignment was moderate $(\mathrm{k}=0.59)$. On PHH3 immunostain, the mitotic figures ranged from 0.1 to $54 / 2 \mathrm{~mm}^{2}$. There is statistically significant but nonlinear correlation between mitoses assessed on $\mathrm{HE}$ and $\mathrm{PHH} 3(\mathrm{p}=0.0001, \mathrm{r}=0.57)$. PHH3 immunostain showed improved intraclass correlation ( single measure $\mathrm{k}=0.92$ ). In addition, there was excellent linear correlation between mitotic rate assessed on $50 \mathrm{HPFs}$ and single hotspot field on PHH3 immunostain $(r>0.97)$. By Kaplan-Meier survival analysis, there was statistically significant survival difference between G1 and G2 tumors as assessed on PHH3 stain (cutoff 3 mitoses $/ 2 \mathrm{~mm}^{2}, \mathrm{p}<0.0001$ ). This cutoff ( $\geq 3$ mitoses $/ 2 \mathrm{~mm}^{2}$ ) corresponds to $\geq 7 / 10 \mathrm{x}$ or $>4 / 20 \mathrm{x}$ hotspot; both also showed statistically significant survival difference between the two grades $(\mathrm{p}<0.0001)$. The Fleiss' kappa correlation for grade assigned by $\mathrm{PHH} 3$ is substantial $(0.8)$

Conclusions: Mitotic rate assessed by $\mathrm{PHH} 3$ stain showed significant correlation with mitotic rate on $\mathrm{HE}$ and improved interobserver reproducibility. Grading based on $\mathrm{PHH} 3$ of either $50 \mathrm{HPF}$ or single hotspot has statistically significant prognostic value, and will provide greater ease of assessment of mitotic rate in pancreatic WDNETs.

1873 Identification of ROS1 Rearrangement in Pancreatic Ductal

\section{Adenocarcinoma}

SM Voss, Z Yang, DS Zander, R Bruggeman, CS Hollenbeak, DM Karamchandani. Penn State Milton S. Hershey Medical Center, Hershey, PA.

Background: The prognosis of pancreatic ductal adenocarcinoma (PDAC) remains poor, and new therapeutic approaches are critically needed. Chromosomal rearrangements in the receptor tyrosine kinase ROS1 have been reported in several neoplasms, most notably non-small cell lung carcinoma (NSCLC). However, the frequency of these rearrangements in PDAC is unknown. NSCLCs harboring ROS1 rearrangements (ROS1 $r$ ) have shown off-target sensitivity to treatment with tyrosine kinase inhibitors (TKIs). Therefore, identification of ROS1 $r$ in PDAC may present a novel opportunity to define a new molecular target enabling responsiveness to TKI therapy.

Design: Tissue microarrays were constructed from archival formalin-fixed, paraffinembedded tissues representing 104 PDACs and 15 non-neoplastic pancreatic controls. Anti-ROS1 immunohistochemistry was performed, and staining was scored based on intensity and percentage of positive cells. Moderate perinuclear staining in at least $50 \%$ of cells, or strong perinuclear staining in at least $10 \%$ of tumor cells, was considered positive. Comparisons between ROS1-positive and -negative groups were performed using a t-test for continuous variables and chi-square test for categorical variables. Results: Six of 104 (5.8\%) PDACs were ROS1-positive. Significant variables associated with ROS1 immunoreactivity included older age at the time of surgery (mean age 76.2 yrs. vs. 66.3 yrs., $\mathrm{p}=0.030$ ), lower $\mathrm{T}$ stage (predominantly $\mathrm{T} 2, \mathrm{p}=0.026$ ), absence of lymphovascular invasion $(\mathrm{p}=0.012)$ and nodal metastases $(\mathrm{p}=0.019)$, and lower TNM stage $(\mathrm{p}<0.0001)$. No significant differences were noted for gender, tumor size, margin involvement, tumor grade, or perineural invasion.

Conclusions: ROS1 $r$ was identified by immunohistochemistry in $5.8 \%$ of PDACs, particularly in lower-stage tumors arising in older patients. This is a significant finding and provides a basis for further studies and clinical trials investigating the clinical effectiveness of ROS1 inhibitors in this distinctive subset of PDACs.

1874 Composite Alterations in Glycolytic Metabolism and Autophagy Are Associated with Poor Prognosis in Pancreatic Ductal Carcinoma

MB Wachsmann, YF Tse, GH Baek, J Mansour, ES Knudsen, A Witkiewicz. University of Texas Southwestern Medical Center, Dallas, TX.

Background: Pancreatic ductal adenocarcinoma (PDA) is one of the most lethal cancers with a 5-year survival rate of $\sim 5 \%$. Pancreatic tumor cells are adept at thriving in a microenvironment of hypoxia and poor nutrient supply. This is achieved through reprogramming of metabolic pathways and stimulating alternative sources for energy. K-RAS mutations occur in $>90 \%$ of PDA and are implicated in promoting glycolytic metabolism and autophagy. However, the metabolic profile of desmoplatic stroma accounting for the majority of PDA volume is considerably less well studied. Here we investigated determinants of glycolysis and autophagy in PDA tumor cells and stroma using a unique combination of clinical specimens and preclinical models.

Design: Immunohistochemistry was performed on tissue microarrays generated from 230 PDA cases. Staining for cleaved LC3 and the monocarboxylate transporter 4 (MCT4) were employed as markers for autophagy and glycolytic metabolism respectively. For LC3, the results were scored according to staining of tumor and stromal cell percentage and were divided into three grades (1) $<20 \%$ of cells, (2) $20-60 \%$ of cells and (3) $>60 \%$ of cells. MCT4 staining in tumor tissue and stroma was scored as previously reported. Overall survival was calculated using the Kaplan-Meier method. Three stromal cell lines and a panel of 12 pancreatic cancer cell lines were used to assay basal autophagy, MCT4 expression, and metabolism. The effect of perturbing these processes on cell viability and apoptosis was determined.

Results: As a single marker cleaved LC3 expression was not associated with overall survival (OS). High MCT4 expression was observed in PDA with shorter OS and cases with high epithelial MCT4 expression and high stromal LC3 expression had the worse prognosis. PDA cell lines and stromal cells exhibit diverse baseline autophagy and metabolic preference. However, stromal cells were particularly sensitive to the inhibition of authophagy with chloroquine. In contrast, specific pancreatic cancer cells were particularly sensitive to glucose withdrawal and the suppression of MCT4 expression.

Conclusions: These data indicate that diverse metabolic processes supporting cell survival are activated in tumor epithelial and stromal compartments. Glycolytic metabolism within the tumor compartment is a particularly important feature of PDA, which is supplemented by effects of autophagy in the tumor microenvironment. Targeting each of these processes could represent an important therapeutic strategy for PDA.

1875 MCT4 Defines a Metabolic Subtype of PDA with Poor Prognosis and Provides Potential Therapeutic Target

$M$ Wachsmann, Y Tse, J Franco, E Knudsen, A Witkiewicz. University of Texas Southwestern Medical Center, Dallas, TX.

Background: Metabolic reprogramming is a hallmark of cancer and targeting tumor specific metabolic dependencies is an emerging therapeutic approach. Due to its genetic features (e.g. K-RAS mutations), tissue architecture, and poor vascularization pancreatic cancer (PDA) has unique metabolic requirements. While there is interest in "cutting off fuel" for PDA, recent studies indicate that there is "metabolic flexibility" allowing cancer cells to use glucose or glutamine depending on the availability of the nutrients in the microenvironment to hinder therapeutic approaches. Therefore, targeting the excretion of "waste" (metabolic by-products from cancer cells) is of interest. In a cohort of cases, we found that PDA characterized by expression of monocarboxylate transporter 4 (MCT4) has particularly poor prognosis. MCT4 facilities export of lactate maintaining intracellular $\mathrm{pH}$ allowing tumors to survive in acidic conditions created by hypoxia and glycolytic metabolism. Little is known regarding the expression of MCT4 in PDA and the impact of targeting MCT4 therapeutically.

Design: MCT4 expression was evaluated in the tumor and stroma compartments of 230 PDA cases. To establish if levels of MCT4 are influenced by the microenviroment we evaluated stromal volume and vessel microdensity (Aperio image analysis system). In parallel, targeted sequencing of PDA cases was performed to identify genetic events that were associated with expression of MCT4. Since MCT4 is involved with intracellular $\mathrm{pH}$ and glycolytic metabolism, MCT4 high and low expressing tumor lines were treated with glycolytic and regulators of $\mathrm{pH}$. To specifically define therapeutic vulnerabilities, a screen of 340 drugs in clinical development was carried out in models with defined levels of MCT4.

Results: KRAS, p53, SMAD4 and mutation in DNA double strand break repair pathway were associated with high levels of MCT4 expression. Additionally, there is a correlative relationship between features of the tumor microenvironment and expression of MCT4. The knockdown of MCT4 had a profound effect on metabolism and lead to the death of PDA cells that highly express MCT4. This supports the concept that MCT4 defines a metabolic subtype of PDA with specific therapeutic vulnerabilities. In keeping with this concept, we defined a number of drugs that were specifically active against PDA models with high expression of MCT4. 
Conclusions: MCT4 expression is associated with specific genetic and microenviromental features of PDA. MCT4 defines a metabolic subtype of PDA with poor prognosis but vulnerabilities that can be therapeutic targets.

1876 Gastrinomas Associated with Multiple Endocrine Neoplasia Type 1 Syndrome: A Clinicopathologic Study of 14 Cases

$B$ Weng, A Rashid, EG Grubbs, JE Lee, H Wang. University of Texas MD Anderson Cancer Center, Houston, TX.

Background: Gastrinoma is the most frequent hormonally-active neuroendocrine tumor (NET) in multiple endocrine neoplasia 1 syndrome (MEN1). It is associated with elevated fasting gastrin serum levels, recurrent peptic ulceration, and occasional diarrhea. In this study, we examined the clinicopathologic features of MEN1-associated gastrinoma.

Design: Our patient population consists of 14 patients with genetically confirmed MEN1 and hypergastrinemia who underwent surgical resection at our institution between 1980 and 2012. Immunohistochemical stain for gastrin was performed on sections from all tumor nodules in the duodenum and pancreas and all positive lymph nodes for all patients. Clinical and pathological information were retrieved from patient medical record.

Results: There were 7 males and 7 females with a mean age 46.1 years (range: $24-59$ years). Median preoperative fasting gastrin level was $1345 \mathrm{pg} / \mathrm{mL}$ (range, 248-7,019). Pancreaticoduodenectomy (PD) was performed in 3 patients, pylorus sparing PD was performed in 8 patients, and distal pancreatectomy was performed in 3 patients. Pancreatic NETs were identified in 13 patients (93\%). Pancreatic NETs were multifoca in 12 patients $(86 \%)$ with a median largest tumor size of $2.1 \mathrm{~cm}$ (range: $0.3-6.5 \mathrm{~cm}$ ). Duodenal NETs were identified in 9/11 (82\%) patients who underwent PD. Among these patients, duodenal NETs were multifocal in 6 patients with the median largest tumo size $1.0 \mathrm{~cm}$ (range: $0.5-2.5 \mathrm{~cm}$ ). Peripancreatic lymph nodes were positive for NET in $92.3 \%$ patients $(12 / 13)$. The median number of positive lymph nodes was 3.5 (range: 1-13). Multifocal gastric NETs were also present in all three patients who underwent PD Duodenal and lymph node metastatic NETs were positive for gastrin in 9/9 $(100 \%)$ and $12 / 12$ patients $(100 \%)$, respectively. In contrast, the pancreatic NETs were negative for gastrin in all patients and none of the gastric NETs were gastrin positive. Postoperative eugastrinemia was achieved in $86 \%$ patients $(12 / 14)$ with a median follow-up of 64 months. All patients were alive with no evidence of disease except one who died from esophageal adenocarcinoma.

Conclusions: Our study demonstrated that MEN1-associated gastrinomas are almost exclusively localized in the duodenum with frequent metastasis to regional lymph nodes. For patients who undergo resection for MEN1-associated gastrinoma, careful examination of the duodenum and regional lymph node dissection should be performed to achieve complete gastrinoma removal.

1877 Correlation between the Ki-67 Labeling Index of Paired Cytology and Resection Specimens from Pancreatic Neuroendocrine Tumors

SE Wobker, KG Greene, LG Dodd. University of North Carolina, Chapel Hill, NC. Background: Neuroendocrine tumors of the pancreas are relatively rare neoplasms. These tumors are often sampled by endoscopic ultrasound-guided fine needle aspiration (FNA) which allows for cytologic evaluation and helps guide clinical management The morphologic features of neuroendocrine differentiation can be appreciated in FNA specimens; however, the role of Ki-67 immunohistochemical (IHC) staining of cell blocks from FNAs is not well-defined. This study is a retrospective review of all pancreatic neuroendocrine tumors with paired FNA and surgical resection specimens identified at UNC between $8 / 1 / 2003$ and $9 / 1 / 2013$. The goal of this study is to determine if there is correlation between the $\mathrm{Ki}-67$ index of paired cytology and resection specimens from pancreatic neuroendocrine tumors.

Design: A CoPath database search is performed to identify instances in which a patient underwent both FNA and subsequent surgical resection at UNC. A diagnosis of pancreatic neuroendocrine tumor in the resection specimen and the presence of a cytology cell block is required for inclusion in the study. Using these criteria, 12 paired specimens are identified. Ki-67 IHC staining is performed on freshly obtained sections from the cell blocks and resections. The Ki-67 index in paucicellular cell blocks is determined by counting the total number of tumor cells present and dividing by the number of positively staining tumor cells. In more cellular specimens, up to 500 cells are evaluated. The Ki-67 index in resection specimens is determined by counting the number of tumor cells present in five $40 x$ fields in the area of greatest staining density and dividing by the number of positively staining tumor cells. The Ki-67 index of each cell block is compared to that of its paired resection specimen to assess for correlation. Results: Of the 12 paired specimens identified, 2 are excluded due to insufficient material in the cell block. Ki-67 indices in the remaining 10 paired specimens show discordant results (Pearson's correlation, $r=0.62$, $p$ value $=0.052$ ). Ki-67 indices in cell blocks ranged from $9.6 \%$ lower than the paired resection specimen to $5.3 \%$ higher. Nine out of 10 cell blocks demonstrated a Ki-67 index greater than $2 \%$, placing these tumors into an intermediate grade category by WHO classification. However, only 5 of the 10 resection specimens demonstrated a Ki-67 index greater than $2 \%$.

Conclusions: Ki-67 IHC staining of cell blocks from FNAs of pancreatic neuroendocrine tumors is not recommended because of a tendency to overestimate the tumor grade.
1878 Analysis of VEGF/PIGF Heterodimer Level in Pancreatic Cyst Fluid as a Biomarker for Serous Cystadenoma

RI Wu, PM Sluss, M Mino-Kenudson, WR Brugge, MB Pitman. Massachusetts General Hospital, Boston, MA.

Background: Pancreatic cyst fluid (PCF) analysis for biochemical and molecular markers provides valuable information in the pre-operative evaluation of pancreatic cysts. Due to the high vascularity of septa in serous cystadenoma (SCA), vascular endothelial growth factor (VEGF) promises to be a marker for identifying SCA in PCF. Design: PCF aspirated from patients during endoscopic ultrasound (EUS) or from resected specimens was analyzed for VEGF/Placenta Growth Factor (PIGF) heterodimer using a double-antibody enzyme-linked immunosorbent assay (ELISA) [RnD Systems (Minneapolis, MN)]. The assay has previously been used for serum or plasma measurements and was validated for PCFs in this study. A positive result was determined to $b e \geq 60 \mathrm{pg} / \mathrm{ml}$. True positive (TP) or false negative (FN) results were determined by histological confirmation of SCA and false positive (FP) or true negative (TN) results with confirmation of a non-SCA cyst by either elevated CEA above $192 \mathrm{ng} / \mathrm{ml}$, elevated amylase above $5000 \mathrm{U} / \mathrm{L}$, or detected KRAS mutation. Statistical analysis was performed using Fisher's exact test at 95\% confidence intervals.

Results: Forty-eight PCFs were analyzed; one was technically inadequate. Of the remaining 47 , only three were positive $(6 \%)$ : one pseudocyst, one cystic adenocarcinoma, and one SCA. Of six histologically confirmed SCAs, only one was positive (17\%). Six PCFs were nonclassifiable due to insufficient data, leaving 41 PCFs for performance calculations ( $33 \mathrm{TN}, 5 \mathrm{FN}, 1 \mathrm{TP}$, and $2 \mathrm{FP}$ ) yielding a sensitivity of $17 \%$ and specificity of $94 \%$

Conclusions: Analysis of PCF for VEGF by ELISA assay of VEGF/PIGF heterodimer appears to an insensitive biomarker for SCA. Although specificity is high by calculations, there are more FP than TP results. Additional study is required to determine clinical utility.

1879 CRABP2 Is a Highly Sensitive and Specific Diagnostic Marker for Pancreatic Ductal Adenocarcinoma

W Xiao, $H$ Hong, A Awadallah, W Xin. University Hospitals Case Medical Center and Case Western Reserve University, Cleveland, $\mathrm{OH}$.

Background: Distinguishing pancreatic ductal adenocarcinoma (PDAC) from other benign pancreatic conditions, such as extensive chronic pancreatitis, can be histologically and cytologically challenging. Molecular biomarkers that have high sensitivity and specificity for PDAC have been rigorously searched with little success. CRABP2, a retinoid acid binding protein, shuffles retinoid acid from cytosol into the nucleus and forms a complex with nuclear retinoid acid receptor to facilitate transcriptional activities of retinoid acid. In this study, we evaluated the expression patterns of CRABP2 in surgical resection and FNA cytology specimens of PDAC. Design: Formalin-fixed paraffin embedded blocks from surgically resected PDAC and cell blocks of FNA pancreatic specimens were extracted from our surgical pathology and cytology archive, respectively. Polyclonal anti-CRABP2 was used and immunohistochemical staining was performed in our hospital diagnostic lab. The control groups include normal pancreatic tissue, chronic pancreatitis and pancreatic intraepithelial neoplasia (PanIN) 1-3

Results: No expressions of CRABP2 were detected in normal pancreatic parenchyma, normal ductal epithelium and chronic pancreatitis. In contrast, intranuclear and cytoplasmic expression of CRABP2 was readily detected in all PDAC $(50 / 50,100 \%$, Fig 1). CRABP2 staining was also observed and progressively increased from PanIN 1 to $3(13.9 \%$ to $88 \%)$. CRABP2 was uniformly negative $(0 / 14)$ in FNA specimens if cytology diagnosis was "benign". When FNA specimens later confirmed as PDAC by resection were evaluated, CRABP2 was positive in $55.6 \%(5 / 9)$ cases if cytology diagnosis was "atypia", and in $87.5 \%$ (14/16) cases if it was "malignancy".

Conclusions: Our study shows that CRABP2 is highly and specifically expressed in PDAC, and is more commonly expressed in high-grade precursor cancerous lesions than in low-grade lesions, and suggests it may involve pancreatic carcinogenesis at earlier stage. CRABP2 could be used as a diagnostic marker to distinguish PDAC from other benign pancreatic conditions in both surgical and FNA cytology specimen. Figure 1. CRABP2 expression in normal pancreas (A), PDAC (B), PanIN (C-D), and FNA specimens (E, chronic pancreatitis; F, PDAC). 


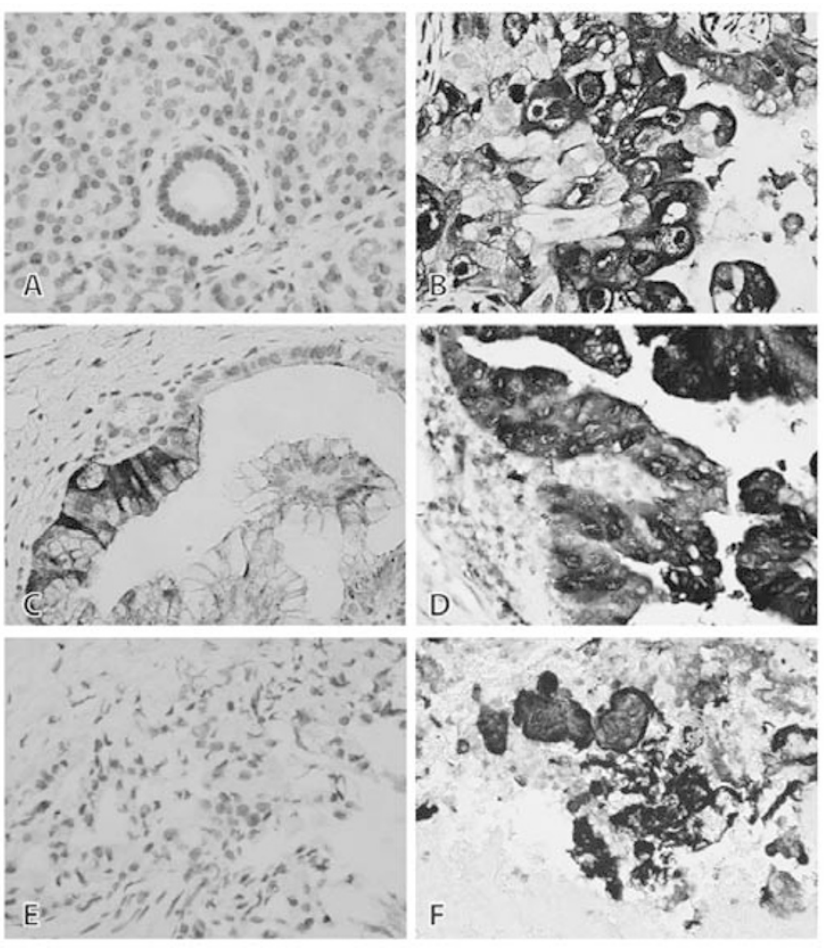

1880 Immunohistochemical Markers and Prognosis with Pancreatic Neuroendocrine Tumors

Z Zhao, MJ Mentrikoski, TW Bauer, JM Lindberg, EB Stelow. University of Virginia Health System, Charlottesville, VA.

Background: Pancreatic neuroendocrine tumors (PanNETs) exhibit a broad range of clinical behavior. However, there are few histological features or other markers to aid pathologists in assessing prognosis. We investigated a series of possible prognostic markers and compared them to outcome for a large series of PanNETs.

Design: 81 cases of resected primary PanNETs were retrieved from our pathology database and the clinical histories reviewed. A tissue microarray was constructed and analyzed with immunohistochemical (IHC) stains, including cytokeratin 19 (CK19), progesterone receptor (PR), CD117, PAX8, Ki-67, HBME1, and CD10. Interpretation of Ki-67 was based on the WHO grading system as 0 (negative), 1 ( $<2 \%$ of nuclei staining), $2(3-20 \%)$ and $3(>20 \%)$. Other markers were considered positive if $>5 \%$ of tumor cells stained.

Results: The IHC results are summarized in the table below.

\begin{tabular}{|c|c|c|c|c|c|c|c|}
\hline & $\begin{array}{l}\text { Ki-67 } \\
\text { index } \\
>2 \%\end{array}$ & CK19 & PAX8 & PR & CD10 & HBME1 & CD117 \\
\hline $\begin{array}{l}\mathrm{LN} \text { metastasis at } \\
\text { surgery }(\mathrm{n}=21)\end{array}$ & $4(19 \%)$ & $\begin{array}{l}13 \\
(62 \%)\end{array}$ & $8(38 \%)$ & $8(38 \%)$ & $4(19 \%)$ & $3(14 \%)$ & $3(14 \%)$ \\
\hline $\begin{array}{l}\text { No LN metastasis at } \\
\text { surgery }(\mathrm{n}=60)\end{array}$ & $5(8 \%)$ & $\begin{array}{l}28 \\
(47 \%)\end{array}$ & $\begin{array}{l}40 \\
67 \%)\end{array}$ & $\begin{array}{l}35 \\
58 \%)\end{array}$ & $\begin{array}{l}13 \\
(22 \%)\end{array}$ & $1(2 \%)$ & $3(5 \%)$ \\
\hline P-value & 0.2279 & 0.3116 & 0.0375 & 0.1324 & 1.000 & 0.0516 & 0.1768 \\
\hline EM at surgery $(n=14)$ & $4(29 \%)$ & $\begin{array}{l}10 \\
(71 \%)\end{array}$ & $5(36 \%)$ & $6(43 \%)$ & $2(14 \%)$ & $2(14 \%)$ & $2(14 \%)$ \\
\hline $\begin{array}{l}\text { No EM at surgery } \\
(\mathrm{n}=67)\end{array}$ & $5(7 \%)$ & $\begin{array}{l}31 \\
(46 \%)\end{array}$ & $\begin{array}{l}43 \\
(64 \%) \\
\end{array}$ & $\begin{array}{l}36 \\
(54 \%)\end{array}$ & $\begin{array}{l}15 \\
(22 \%)\end{array}$ & $3(4 \%)$ & $4(6 \%)$ \\
\hline P-value & 0.0435 & 0.1405 & 0.0721 & 0.5612 & 0.7227 & 0.2043 & 0.2759 \\
\hline Recurrence $(\mathrm{n}=17)$ & $3(18 \%)$ & $\begin{array}{l}10 \\
(59 \%)\end{array}$ & $7(41 \%)$ & $\begin{array}{l}10 \\
(59 \%)\end{array}$ & | $3(18 \%)$ & $2(12 \%)$ & $2(12 \%)$ \\
\hline No recurrence $(\mathrm{n}=64)$ & $6(9 \%)$ & $\begin{array}{l}30 \\
(47 \%)\end{array}$ & $\begin{array}{l}40 \\
(63 \%)\end{array}$ & $\begin{array}{l}32 \\
(50 \%)\end{array}$ & $\left.\begin{array}{l}13 \\
(20 \%)\end{array}\right)$ & $3(5 \%)$ & $4(6 \%)$ \\
\hline P-value & 0.3877 & 0.4241 & 0.1666 & 0.5917 & 1.000 & 0.2808 & 0.6006 \\
\hline $\begin{array}{l}\text { No metastasis at surgery } \\
\text { with recurrence }(n=9)\end{array}$ & $1(11 \%)$ & $4(44 \%)$ & $\mid 5(56 \%)$ & $6(67 \%)$ & $2(22 \%)$ & $0(0 \%)$ & $0(0 \%)$ \\
\hline $\begin{array}{l}\text { No metastasis at surgery } \\
\text { and no recurrence } \\
(\mathrm{n}=44)\end{array}$ & $4(9 \%)$ & \begin{tabular}{|l}
19 \\
$(43 \%)$
\end{tabular} & $\begin{array}{l}32 \\
(73 \%)\end{array}$ & $\begin{array}{l}26 \\
(59 \%)\end{array}$ & $\begin{array}{l}10 \\
(23 \%)\end{array}$ & $2(5 \%)$ & $3(7 \%)$ \\
\hline P-value & 1.000 & 1.000 & 0.4274 & 1.000 & 1.000 & 1.000 & 1.000 \\
\hline
\end{tabular}

More cases with extranodal metastasis (EM) at surgery compared to those without had recurrent disease ( $50 \mathrm{vs} 15 \%, \mathrm{p}=0.0077$ ), but LN metastasis alone at surgery did not predict recurrent disease ( 33 vs $17 \%, \mathrm{p}=0.1262$ ). Importantaly, no marker predicted recurrent disease for tumors without metastasis (stage I and IIA) at surgery.

Conclusions: Our study confirmed previous studies, showing tumor grade, Ki-67 index, PAX8 and HBME1 staining predict stage at time of resection. Presence of EM at surgery is predictive of disease recurrence. Nonetheless, these markers, including the Ki-67 index, seem to be of limited use for predicting which stage I and IIA PanNETs will eventually recur.

\section{Pathobiology}

1881 Discriminating Uterine Serous Carcinoma from High Grade Ovarian and Fallopian Tube Serous Carcinoma by Whole Genome Expression QF Ahmed, S Bandyopadyay, B Alosh, E AbdulFatah, S Barak, G Dyson, A BolligFischer, RAli-Fehmi. Wayne State University, Detroit, MI; Karmanos Cancer Institute, Detroit, MI.

Background: The morphologic and molecular similarities amongst uterine serous carcinoma (USC), high grade ovarian serous carcinoma (OSC) and fallopian tube serous carcinoma (FTSC) are well known. The aim of our study was to analyze and highlight the differences at the molecular level.

Design: We retrieved 24 cases of primary high grade OSC $(\mathrm{n}=8), \operatorname{FTSC}(\mathrm{n}=8)$ and USC $(\mathrm{n}=8)$ age and stage matched from our pathology database. We measured whole-genome mRNA expression in all formalin-fixed paraffin (FFPE) specimens. Total RNA was isolated and RNA quantity was estimated using a NanoDrop 1000 spectrophotometer. Further sample processing and gene expression profiling was done using an Illumina Human Whole-Genome HT Assay kit. A total of 29,000 annotated genes derived from RefSeq were measured. A t-test determined significant differential gene expression. Results: High grade (OSC) and (FTSC) did not show any significant difference in mRNA expression supporting the concept of similar cell of origin and development pathway in both. At the same time USC was distinct. 1,740 genes were differentially expressed $(\mathrm{p}<.05)$ for both uterine versus ovarian and uterine versus fallopian cancer comparisons. A pathway impact analysis of the resulting gene set indicated significant pathway-level differences, listed in Table 1.

\begin{tabular}{|c|c|c|c|c|c|}
\hline Pathway name & $\begin{array}{l}\text { Impact } \\
\text { factor }\end{array}$ & $\begin{array}{l}\text { \#Genes in } \\
\text { pathway }\end{array}$ & \begin{tabular}{|l} 
\#Input genes \\
in pathway
\end{tabular} & $\begin{array}{l}\text { \#Pathway } \\
\text { genes on } \\
\text { array }\end{array}$ & p-value \\
\hline $\begin{array}{l}\text { Cell adhesion molecules } \\
\text { (CAMs) }\end{array}$ & 122.391 & 134 & 22 & 134 & 2.40E-04 \\
\hline $\begin{array}{l}\text { Leukocyte transendothelial } \\
\text { migration }\end{array}$ & $\mid 06.673$ & 119 & 20 & 119 & |3.29E-04 \\
\hline $\begin{array}{l}\text { Antigen processing and } \\
\text { presentation }\end{array}$ & $41 / 528$ & 89 & 3 & 89 & 0.960 \\
\hline ECM-receptor interaction & 27.573 & 84 & 27 & 84 & $5.00 \mathrm{E}-12$ \\
\hline $\begin{array}{l}\text { Phosphatidylinositol/PLC } \\
\text { signaling system }\end{array}$ & $\mid 26.366$ & 76 & $\mid 10$ & 76 & 0.046 \\
\hline Focal adhesion & 25.203 & 203 & 43 & 203 & $1.21 \mathrm{E}-10$ \\
\hline Calcium signaling pathway & 11.25 & 182 & 27 & 182 & $2.85 \mathrm{E}-04$ \\
\hline Gap junction & 10.625 & 96 & 16 & 96 & 0.001 \\
\hline Tight junction & 10.411 & 135 & 22 & 135 & $2.67 \mathrm{E}-04$ \\
\hline GnRH signaling pathway & 7.068 & 103 & 16 & 103 & 0.003 \\
\hline Adherens junction & 6.924 & 78 & 11 & 78 & 0.024 \\
\hline TGF-beta signaling pathway & 6.758 & 87 & 13 & 87 & 0.009 \\
\hline Insulin signaling pathway & 6.735 & 138 & 19 & 138 & 0.005 \\
\hline MAPK signaling pathway & 6.527 & 272 & 30 & 272 & 0.014 \\
\hline
\end{tabular}

Table 1. Enriched pathways and their significance according to pathway level analysis using Ontotool pathway express

Conclusions: Understanding molecular differences between these diseases might help in identifying some of the clinical differences, prognostic biomarkers and targeted treatment to improve USC outcomes.

1882 Progesterone Induces NF-kB Activation through PI3K/Akt-2 Signal Pathway Dependent of Heterotrimeric Gi/Go Proteins in MCF-7 Breast Cancer

F Candanedo-Gonzalez, P Cortes-Reynosa, N Serna-Marquez, S Villegas-Comonfort, R Espinoza-Neira, A Soto-Guzman, E Perez-Salzar. CINVESTAV, Mexico City, DF, Mexico.

Background: Around $75 \%$ of breast tumors are positive for the progesterone receptor (PR). Less than $10 \%$ of estrogen receptor-negative (ER-) breast cancers are progesterone receptor-positive $(\mathrm{PR}+)$, which most often affects women under 50 years of age with high-grade tumors with more aggressive behavior. However, the signal transduction pathways activated by progesterone have not been studied in detail.

Design: Objective: To better understand the role of the rapid non-genomic signalling effects of progesterone, we investigated the mechanisms leading to NF-kB activation. Methods: MCF-7 and MDA-MB-231 breast cancer cells were cultured in DMEM and were serum-starved for $12 \mathrm{~h}$ before treatment with inhibitors (PTX, Wortmaninn, Rapamycin, A6730 Akt-1/2 kinase inhibitor, PP2, PP3) and/or progesterone (100 nM) for $30 \mathrm{~min}$ and evaluated by Western blot. Statistical analysis: Results are expressed as mean $\pm \mathrm{SD}$. Data was statistically analyzed using one-way ANOVA. Statistical probability of $\mathrm{p}<0.05$ was considered significant.

Results: Stimulation of MCF-7 cells with progesterone induced the rapidly NF-kB activation, in a time and concentration-dependent manner. The inhibition with PTX, Wortmaninn, Ag730 and PP2 completely prevent the NF-kB activation. These results showed that the mechanism of NF-kB activation induced by progesterone is dependent of heterotrimeric Gi/Go proteins, c-Src, and PI3K/Akt-2 signaling pathways.

Conclusions: This is the first study to show that progesterone stimulates the NF-kB activation through PI3K/Akt-signal pathway dependent of heterotrimeric Gi/Go proteins in MCF-7 breast cancer cells. Understanding the role of the progesterone and its receptor in breast cancer, will allow to offer new therapeutic options in the future. 